\title{
Nonlinear corrections in basic problems of electro- and magneto-statics in the vacuum.
}

\author{
Caio V. Costa ${ }^{1 *}$, Dmitry M. Gitman ${ }^{1 \dagger}$ and Anatoly E. Shabad ${ }^{2 \ddagger}$ \\ ${ }^{1}$ Instituto de Física, Universidade de São Paulo, \\ Caixa Postal 66318, CEP 05508-090, São Paulo, S. P., Brazil \\ ${ }^{2}$ P. N. Lebedev Physics Institute, Leninsky Prospekt 53, Moscow 117924, Russia
}

August 25, 2018

\begin{abstract}
We find third-power nonlinear corrections to the Coulomb and other static electric fields, as well as to the electric and magnetic dipole fields, as we work within QED with no background field. The nonlinear response function we base our consideration on is the fourth-rank polarization tensor, calculated within the local (infrared) approximation of the effective action. Therefore, the results are applicable to weakly varying fields. It is established that the nonlinear correction to magnetic moment of some baryons just matches, in the order of magnitude, the existing gap between its experimental and theoretical values.
\end{abstract}

\section{Introduction}

Quantum electrodynamics (QED) is a nonlinear theory that includes effective interaction between electromagnetic fields realized by creation of virtual pairs of charged particles, electrons and positrons, that interact with the electromagnetic fields before annihilating. The nonlinearity becomes usually essential when the electromagnetic fields involved in the problem reach and exceed the characteristic "Schwinger's" value of the order of $m^{2} / e$, where $m$ and $e$ are electron mass and charg $€^{1}$. In the Gauss system of units this value is $B_{\mathrm{Sch}}=4.4 \times 10^{13} \mathrm{G}$ for magnetic and $E_{\mathrm{Sch}}=1.3 \times 10^{16} \mathrm{~V} / \mathrm{cm}$ for electric field, while in the Heaviside-Lorenz (HL) system, mostly used in the present paper, these values are $1.2 \times 10^{13} \mathrm{G}$ and $0.37 \times 10^{16} \mathrm{~V} / \mathrm{cm}$.

Apart from the customary reference to sufficiently large magnetic fields that existed in the early Universe [5] or are existing 4, 6 in pulsars and magnetars (to be discussed in Section 5 below), it is lately noted that the electric fields above Schwinger's value are expected [7] to occur in quark stars, and that very strong magnetic field is also formed for a short time when accelerated charged particles (heavy ions) collide provided that the impact parameter is nonzero 8 . Once the magnetic field arising in the collision is of the order of hadron mass-squared and is thus apt of interfering with strong interactions, this circumstance already avoked a vivid activity in QCD [9, lattice calculations [10] included.

Presently, we have also to note that large electric and magnetic fields may be associated with baryons, due to their charges and/or to their electric and magnetic multipole moments. Generally, large fields occur in the vicinity of charged elementary particles or paricles carrying a magnetic or electric moment, or both. Where these are protons, neutrons and, generally, atomic nuclei, the stemming nonlinearity may affect the atomic spectra. (Within a nonlinear electrodynamics theory, other than QED, namely, the noncommutative $U_{\star}(1)$-gauge theory, this issue was touched in 11.) Consider, for instance, the neutron, whose magnetic moment $\mathcal{M}$ is of the order of 2 nuclear magnetons $\mu_{N}=5.05 \cdot 10^{-24} \mathrm{~g}^{1 / 2} \mathrm{~cm}^{5 / 2} \mathrm{~s}^{-1}$. The magnetic dipole field at the distance $r$ is, at its maximum, $\sim 2 \mathcal{M} / r^{3}$. It reaches Schwinger's value $4.4 \cdot 10^{13} \mathrm{~g}^{1 / 2}$ $\mathrm{cm}^{-1 / 2} \mathrm{~s}^{-1}$ at the distance an order of magnitude larger than the neutron magnetic size of $\sim 1 \mathrm{fm}$, therefore the nonlinear correction to the nucleon magnetic moment interacion with the orbital electron comes into play at the distance of about five thousandth of the Bohr radius, which may be significant in the atomic scale.

\footnotetext{
*Electronic address: caiocostalopes@usp.br

$\dagger$ Electronic address: gitman@dfn.if.usp.br

${ }^{\ddagger}$ Electronic address: ashabad@Ipi.ru

${ }^{1}$ The only known exception is provided by the resonance [1] in the vacuum polarization responsible for the capture of the photon 2$]$ in pulsar magnetospheres, where the photon forms a mixed state with the mutually bound $\mathrm{e}^{+} \mathrm{e}^{-}$pair [3] 4. This effect is essential at the magnetic field values of already $\approx 0.1 B_{\mathrm{Sch}}$.
} 
There is a vast literature on nonlinear effects in QED, where the electromagnetic field, which is necessary to be taken as large is the so called external field that forms the vacuum background, see, e.g., the monographs [12, 13, 14. In this approach, a great advantage of going beyond the perturbation expansions in powers of the external field is achieved thanks to the use of special fields that admit exact solutions for the Dirac electron Green function, like fields constant in space and time and the plane wave (laser) field. In the present, as well as in the previous [15, paper we are interested also in another class of fields, namely those that are produced by spherically and cylindrically symmetric static sources, including the fields of electric and magnetic dipole. As far as exact Dirac solutions for such fields are either unknown or overcomplicated to exploit, we treat them relying on the power expansion and take into account the lowest nontrivial power. Their strength should be enough to make the contribution of nonlinearity significant, but still sufficiently small to keep below the value, where the power expansion remains meaningful. On this basis we considered previously the magnetic field produced by a static charge placed in a strong constant and homogeneous magnetic field [15. The latter was treated as the nonperturbative background, it might exceed the Schwinger value, whereas the contribution of the static charge was retained up to the second power. The very existence of this contribution (the magneto-electric effect in QED) is provided by the three-photon diagram (third-rank polarization tensor) off the photon mass shell, nonzero against the external background. Now we switch off the background field and confine ourselves to various static sources in the blank vacuum. This time, the third-rank polarization tensor vanishes due to the C-invariance, and the first nonlinear self-coupling of the sources originates from the fourth-order polarization tensor associated with the four-photon diagram. This diagram, when reduced to the mass shell of four or two photons, describes the photon-by-photon scattering and scattering of a photon off a Coulomb center, respectively. This exhausts all scattering processes with the four-prong diagram [16. However, when all the four photon legs, are taken off-shell, i.e., beyond the photon dispersion curves, this diagram can be used for calculation of self-couplings of various sources of electromagnetic fields. We present a framework for considering slowly varying in time and space sources on the basis of effective action formalism, where the fourth-rank polarization tensor is calculated by four variational field-derivatives of the effective action taken as a local functional of the field invariants. This corresponds, in momentum space, to the infrared limit of small 4-momenta. This framework supplies us with the simplest possible approximation, wherein the self-coupling becomes first pronounced. For a point-like electric charge the nonlinear correction to the Coulomb field presented in [17] (commented on also in [15]) is reproduced. Extended electric and magnetic sources of various symmetries are studied, and nonlinear corrections to the fields produced by them are found, the most attention paid to the electric and magnetic dipole fields. These fields reproduce themselves under the mapping due to the nonlinearity, while the resulting magnetic and electric moments undergo nonlinear corrections, becoming subject of cubic equations.

Our numerical estimates show that the fields of giant magnetic dipoles, the pulsars [18, do produce essential nonlinear corrections, before they reach the values of the order of $10^{15} \mathrm{G}$, when we are already taken out of the scope of validity of our power expansion. The nonlinear corrections can hardly be distinguished by a far-remote observer, since these lead only to renormalization of the magnetic moment. However, inside and in the close vicinity of magnetars the nonlinear corrections to any theoretically derived model values of the magnetic field are huge, and must be taken into account when considering various physical processes in these regions.

As far as baryons are concerned we find that the magnetic moment of the neutron is too large to be treated within the present power-expansion approach. Nevertheless, the nonlinear corrections to smaller magnetic moments of some other baryons do fit this approach and prove to be of the same order of magnitude as the existing indeterminacy in the theoretical results for their magnetic moment values calculated within the theory of strong interactions. This means that the nonlinearity of QED will have to be taken into account already at the very next step of perfection of the theoretical predictions for the magnetic moments of these neutral baryons.

Before proceeding, we find it interesting to note in passing that the magnetization of the order of extreme magnetars may be associated with the magnetic moment of the neutron. If one imagines a neutron star as composed of neutrons in a ferromagnetic state, i.e. with all magnetic moments of the constituent neutrons parallel to each other, and packed as densely as their electromagnetic size permits, the magnetization of that star would be the same as the magnetization of an individual neutron, defined as its magnetic moment per unit volume. This is about (see Subsection 5.2 below) $3 \times 10^{16}$ G. This numerical observation may support the idea of essential nucleon contribution (developed, e.g., in [19]) into the magnetic field of neutron stars (besides the notion of electron currents) and of the magnetar value.

The paper is organized as follows. In Section 2 for any U(1)- gauge-invariant P-even theory we present the nonlinear Maxwell equations for field potentials to the fourth-power accuracy in gauge fields . The second-, third- and fourth-rank polarization tensors are calculated in Appendix1 by differetiations of the effective action over the external constant field to be set equal to zero afterwards. When there is no background, only the third-power nonlinear vacuum response, contained in the fourth-order field-derivative of the effective action, survives the infrared limit. The quadratic nonlinear response is absent, as noted above. As for the linear response, it is trivial in the zero-momentum limit: the second-rank polarization tensor vanishes due to the correspondence principle. We define a nonlinearly induced current as the source of the nonlinear correction to the fields.

In Section 3 we derive a representation for the nonlinear field strengths in special cases where either only magnetic or electric field is present. These representations contain projection operators. In Section 4, Subsection 4.1, the highly 
symmetric configurations are considered, for which these projection operators are identities, and the fields are mapped to their nonlinear counterparts in a local way. In this context nonlinear corrections to spherically symmetric extended charges, charged thread, charged plane of finite thickness and a wire with current are found. More complicated cylindrically symmetric dipole fields produced by a current and by a charge distributed in a special way over the surface of a sphere are studied in Subsection 4.2. The details of calculations intended to realize the projection operators are given in Appendix 2 (magnetic dipole) and Appendix 3 (electric dipole). The resulting equations for self-coupling of dipole moments are discussed. We found that there is no spontaneous creation of these quantities thanks to special property of the second derivative of the effective action that reflects the causality and unitarity properties of the effective action. At the last step the Euler-Heisenberg (local) effective action is used to specify the results dynamically to QED.

In Section 5 we make numerical estimates of the results as applied to magnetic stars and baryons. The contents is summarized in concluding Section 6 .

We adhere to the rationalized Heaviside system of units with $\hbar=c=\varepsilon_{0}=1$ throughout, unless the opposite is indicated.

\section{Nonlinear electromagnetic field equations}

We describe the effective action

$$
\Gamma=\int \mathfrak{L}(z) \mathrm{d}^{4} z
$$

in QED as the Legendre transform of the generating functional of the Green functions [20]. It is, in its turn, the generating functional of the one-particle-irreducible vertices. Eq. (1) is a nonlocal functional, $\mathfrak{L}$ being the effective Lagrangian, which depends on the relativistic field invariants $\mathfrak{F}(x)=\frac{1}{4} F_{\mu \nu}(x) F^{\mu \nu}(x), \mathfrak{G}(x)=\frac{1}{4} F_{\mu \nu}(x) \tilde{F}^{\mu \nu}(x)$ and, generally, on their space-time derivatives of any order. Here the field strength is $F_{\mu \nu}(x)=\partial_{\mu} A_{\nu}(x)-\partial_{\nu} A_{\mu}(x)$, with $A_{\mu}(x)$ being the field potentials, and $\tilde{F}_{\mu \nu}(x)=\frac{1}{2} \epsilon_{\mu \nu \alpha \beta} F^{\alpha \beta}(x)$ is the dual tensor, where $\epsilon_{\mu \nu \alpha \beta}$ is the fully antisymmetric Levi-Civita tensor, such that $\epsilon_{0123}=1$. The Greek indices span the 4-dimensional Minkowski space taking the values $0,1,2,3$. The metric tensor is $\left(\eta_{\mu \tau}\right)=\operatorname{diag}(1,-1,-1,-1)$, and latin indices take the values $1,2,3$.

Beyond QED the action (1) should be thought of a primarily given classical action of a nonlinear theory, e.g., the Born-Infeld action or its nonlocal generalization. As a matter of fact the contents of this article, down to the very last step, where we refer to the Euler-Heisenberg Lagrangian, is independent of special dynamcs: it covers QED as well as any other nonlinear electrodynamcs.

Once we want to find and solve the Euler-Lagrange equations, which the potentials $A^{\mu}(x)$ must satisfy, given some current $J_{\beta}(x)$, then we need to apply the variational principle to the total action $S_{t o t}$, defined as

$$
\begin{aligned}
S_{\text {tot }} & =S+S_{\text {int }}, S=S_{\text {free }}+\Gamma \\
S_{\text {free }} & =-\int \mathfrak{F}(z) \mathrm{d}^{4} z, S_{\text {int }}=-\int J_{\beta}(z) A^{\beta}(z) \mathrm{d}^{4} z .
\end{aligned}
$$

The variational principle imposes minimum action on $S_{\text {tot }}$, i.e., $\frac{\delta S_{\text {tot }}}{\delta A^{\beta}(x)}=0$, therefore we get the Euler-Lagrange equations

$$
J_{\mu}(z)=\frac{\delta S_{\text {free }}}{\delta A^{\mu}(x)}+\frac{\delta \Gamma}{\delta A^{\mu}(x)} .
$$

They provide exact nonlinear equations for the c-valued electromagnetic field that include all quantum corrections inherent in QED. The linear part of these equations makes the standard Maxwell equations, valid in the small-field limit, in an equivalent medium formed by the polarized vacuum with or without external field background.

Note that when writin Eqs. (2), (3) we keep to HL system, since the energy of of the free field and the Euler-Lagrange (Maxwell) equations do not contain the factor $1 / 4 \pi$.

\subsection{Expansion in powers of the field above the background}

Subdivide the current in two parts: $J_{\beta}(x)=j_{\beta}(x)+\mathcal{J}_{\beta}(x)$, where $\mathcal{J}_{\beta}(x)$ is the current supporting an external field $\mathcal{A}^{\beta}(x)$ via the exact nonlinear Maxwell equation $\mathcal{J}_{\beta}(x)=\left.\frac{\delta S}{\delta A^{\beta}(x)}\right|_{A=\mathcal{A}}=\left[\square \eta_{\beta \tau}-\partial^{\beta} \partial^{\tau}\right] \mathcal{A}^{\tau}(x)+\left.\frac{\delta \Gamma}{\delta A^{\beta}(x)}\right|_{A=\mathcal{A}}$. We shall seek solutions in the form $A^{\beta}(x)=\mathcal{A}^{\beta}(x)+a^{\beta}(x)$. The current $j_{\beta}(x)$ is supposed to be small, and then we can expand 
$\frac{\delta \Gamma}{\delta A^{\beta}(x)}$ in powers of the small field $a^{\beta}(x)$ above the external field background. Hence, (3) becomes

$$
\begin{aligned}
& j_{\mu}(x)=\left[\square \eta_{\mu \tau}-\partial_{\mu} \partial_{\tau}\right] a^{\tau}(x)+\int \mathrm{d}^{4} y \Pi_{\mu \tau}(x, y) a^{\tau}(y) \\
& +\frac{1}{2} \int \mathrm{d}^{4} y \mathrm{~d}^{4} u \Pi_{\mu \tau \sigma}(x, y, u) a^{\tau}(y) a^{\sigma}(u) \\
& +\frac{1}{6} \int \mathrm{d}^{4} y \mathrm{~d}^{4} u \mathrm{~d}^{4} v \Pi_{\mu \tau \sigma \rho}(x, y, u, v) a^{\tau}(y) a^{\sigma}(u) a^{\rho}(v),
\end{aligned}
$$

where $\square=\partial_{0}^{2}-\nabla^{2}$, and we have restricted ourselves to the third-power terms in the expansion. The expansion parameter can be revealed no sooner than the matrix coefficients of the expansion (2-, 3- and 4-rank polarizatioin tensors)

$$
\begin{gathered}
\Pi_{\mu \tau}\left(x, x^{\prime}\right)=\left.\frac{\delta^{2} \Gamma}{\delta A^{\mu}(x) \delta A^{\tau}\left(x^{\prime}\right)}\right|_{A=\mathcal{A}}, \\
\Pi_{\mu \tau \sigma}\left(x, x^{\prime}, x^{\prime \prime}\right)=\left.\frac{\delta^{3} \Gamma}{\delta A^{\mu}(x) \delta A^{\tau}\left(x^{\prime}\right) \delta A^{\sigma}\left(x^{\prime \prime}\right)}\right|_{A=\mathcal{A}} \\
\Pi_{\mu \tau \sigma \rho}\left(x, x^{\prime}, x^{\prime \prime}, x^{\prime \prime \prime}\right)=\left.\frac{\delta^{4} \Gamma}{\delta A^{\mu}(x) \delta A^{\tau}\left(x^{\prime}\right) \delta A^{\sigma}\left(x^{\prime \prime}\right) \delta A^{\rho}\left(x^{\prime \prime \prime}\right)}\right|_{A=\mathcal{A}}
\end{gathered}
$$

are calculated within one or another dynamical scheme. The polarization tensors of every $\operatorname{rank} \Pi_{\mu \tau \ldots \sigma}\left(x, x^{\prime}, \ldots x^{\prime \prime}\right)$ satisfy the continuity relations with respect to every argument and every index as a consequence of the gauge invariance.

In this work, we shall mainly deal with external fields $\mathcal{F}_{\alpha \beta}=\partial^{\alpha} \mathcal{A}_{\beta}^{\text {ext }}-\partial^{\beta} \mathcal{A}_{\alpha}^{\text {ext }}$ equal to zero and call this background the blank vacuum. (The only exceptions are calculations in Appendix 1, which include the field $\mathcal{F}_{\alpha \beta}$ constant in space and time within the framework explained in [15]). In this case (also for the constant background) all-rank polarization tensors depend on their coordinate differences. Eq. (4) is the field equation for small electromagnetic perturbations $a^{\beta}(x)=A^{\beta}(x)-\mathcal{A}^{\beta}(x)$ over the blank vacuum, caused by a small external current $j_{\rho}(x)$ and taken to the lowest-power nonlinearity.

With the definition of the photon propagator $D_{\mu \nu}\left(x, x^{\prime}\right)$

$$
D_{\mu \nu}^{-1}\left(x-x^{\prime}\right)=\left[\eta_{\mu \nu} \square-\partial^{\mu} \partial^{\nu}\right] \delta^{(4)}\left(x^{\prime}-x\right)+\Pi_{\mu \nu}\left(x-x^{\prime}\right)
$$

the nonlinear field equations (4) take the form of (the set of) integral equations

$$
\begin{gathered}
a^{\lambda}(x)=\int d^{4} y D^{\lambda \rho}(x-y) j_{\rho}(y)+\int d^{4} y D^{\lambda \rho}(x-y) j_{\rho}^{\mathrm{nl}}(y), \\
j_{\mu}^{\mathrm{nl}}(x)=-\frac{1}{2} \int \mathrm{d}^{4} y \mathrm{~d}^{4} u \Pi_{\mu \tau \sigma}(x, y, u) a^{\tau}(y) a^{\sigma}(u) \\
-\frac{1}{6} \int \mathrm{d}^{4} y \mathrm{~d}^{4} u \mathrm{~d}^{4} v \Pi_{\mu \tau \sigma \rho}(x, y, u, v) a^{\tau}(y) a^{\sigma}(u) a^{\rho}(v),
\end{gathered}
$$

where we define $j_{\mu}^{\mathrm{nl}}(x)$, and call it "nonlinearly induced current".

\subsection{Infrared approximation and its application in no-external-field vacuum}

From now on we shall restrict ourselves only to slowly varying fields $a^{\lambda}(x)$ and, correspondingly, to consideration of the sources $j_{\rho}(y)$ that give rise to such fields via equations (9), (10). To this end we may take the effective action $\Gamma$ in the local limit, where the field derivatives are disregarded from this functional. We also call this limit infrared, because the variational derivatives for the $n$-rank polarization operators (5), (6), (7) become in the local limit their low-momentum asymptotes $\sim k^{n}$, where the momentum $k_{\mu}$ is the variable, Fourier-conjugate to $x_{\mu}$. The resulting expressions for (5), (6), (77) with the background fields $\mathcal{F}_{\alpha \beta}=\partial^{\alpha} \mathcal{A}_{\beta}^{\text {ext }}-\partial^{\beta} \mathcal{A}_{\alpha}^{\text {ext }}$ being arbitrary combinations of constant and homogeneous electric and magnetic fields are calculated in the infrared limit following the same method as in [15, and listed in Appendix 1, Eqs. (83), (84), (85). Henceforward, however, we set the background field $\mathcal{F}_{\alpha \beta}$ equal to zero everywhere, because we are interested only in the blank vacuum as the background medium in the present work, leaving the $\mathcal{F}_{\alpha \beta} \neq 0$ calculations as a billet for a future use. Then the variational derivatives in (5), (6) and (7) are expressed in terms of derivatives of $\mathfrak{L}(\mathfrak{F}, \mathfrak{G})$ with respect to the field invariants reduced to the null external field $\mathcal{A}=0$.

Define $\mathfrak{L}_{\mathfrak{F}}=\left.\frac{\partial \mathfrak{L}}{\partial \mathfrak{F}}\right|_{\mathfrak{F}=0, \mathfrak{G}=0}, \mathfrak{L}_{\mathfrak{G}}=\left.\frac{\partial \mathfrak{L}}{\partial \mathfrak{G}}\right|_{\mathfrak{F}=0, \mathfrak{G}=0}, \mathfrak{L}_{\mathfrak{F} \mathfrak{F}}=\left.\frac{\partial^{2} \mathfrak{L}}{\partial \mathfrak{F}^{2}}\right|_{\mathfrak{F}=0, \mathfrak{G}=0}, \mathfrak{L}_{\mathfrak{F} \mathfrak{G}}=\left.\frac{\partial^{2} \mathfrak{L}}{\partial \mathfrak{F} \partial \mathfrak{G}}\right|_{\mathfrak{F}=0, \mathfrak{G}=0}$ and $\mathfrak{L}_{\mathfrak{G} \mathfrak{G}}=\left.\frac{\partial^{2} \mathfrak{L}}{\partial \mathfrak{G}{ }^{2}}\right|_{\mathfrak{F}=0, \mathfrak{G}=0}$ The relations $\mathfrak{L}_{\mathfrak{F}}=\mathfrak{L}_{\mathfrak{G}}=0$ hold thanks to the correspondence principle, that reads that in the infrared limit the standard 
linear Maxwell equations for the no-background-field case should be exact, not subject to any corrections. Another reason for $\mathfrak{L}_{\mathfrak{G}}$ (also for $\mathfrak{L}_{\mathfrak{F} \mathfrak{G}}$ ) to vanish is the P-invariance. Setting $\mathcal{F}_{\alpha \beta}=\tilde{\mathcal{F}}_{\alpha \mu}=0$ in (83), (84), (85) we make sure that the linear response in the blank vacuum is trivial in the local (infrared) limit2 (and not beyond it, of course):

$$
\Pi_{\mu \tau}(x, y)=0
$$

while the quadratic response disappears:

$$
\Pi_{\mu \tau \sigma}(x, y, u)=0,
$$

the latter property being as a matter of fact a consequence of C-invariance, valid beyond the infrared approximation as well. As for the cubic response, it is governed by the fourth-rank tensor (7), which in the current case follows from (85) to be

$$
\begin{aligned}
& \Pi_{\mu \tau \sigma \rho}(x, y, u, v)= \\
& =\int \mathrm{d}^{4} z\left\{\mathcal{P}_{\alpha \beta \gamma \lambda \mu \tau \sigma \rho} \frac{\partial \delta^{4}(x-z)}{\partial z_{\alpha}} \frac{\partial \delta^{4}(y-z)}{\partial z_{\beta}} \frac{\partial \delta^{4}(u-z)}{\partial z_{\gamma}} \frac{\partial \delta^{4}(z-v)}{\partial z_{\lambda}}\right\},
\end{aligned}
$$

where we define the constant tensor $\mathcal{P}_{\alpha \beta \gamma \lambda \mu \tau \sigma \rho}$

$$
\begin{aligned}
& \mathcal{P}_{\alpha \beta \gamma \lambda \mu \tau \sigma \rho}=\mathfrak{L}_{\mathfrak{F} \mathfrak{F}}\left[\left(\eta_{\alpha \lambda} \eta_{\rho \mu}-\eta_{\mu \lambda} \eta_{\alpha \rho}\right)\left(\eta_{\tau \sigma} \eta_{\beta \gamma}-\eta_{\beta \sigma} \eta_{\tau \gamma}\right)\right. \\
& \left.+\left(\eta_{\beta \lambda} \eta_{\rho \tau}-\eta_{\tau \lambda} \eta_{\beta \rho}\right)\left(\eta_{\mu \sigma} \eta_{\alpha \gamma}-\eta_{\alpha \sigma} \eta_{\mu \gamma}\right)+\left(\eta_{\gamma \lambda} \eta_{\rho \sigma}-\eta_{\sigma \lambda} \eta_{\gamma \rho}\right)\left(\eta_{\mu \tau} \eta_{\alpha \beta}-\eta_{\mu \beta} \eta_{\alpha \tau}\right)\right] \\
& +\mathfrak{L}_{\mathfrak{G G}}\left[\epsilon_{\alpha \mu \beta \tau} \epsilon_{\lambda \rho \gamma \sigma}+\epsilon_{\lambda \rho \alpha \mu} \epsilon_{\beta \tau \gamma \sigma}+\epsilon_{\lambda \rho \beta \tau} \epsilon_{\alpha \mu \gamma \sigma \sigma}\right] .
\end{aligned}
$$

When deriving this expression we have restricted ourselves to the P-even theories, to which class QED belongs, by imposing the extra condition $\mathfrak{L}_{\mathfrak{F} \mathfrak{G}}=0$. Integrating (13) by parts we obtain

$$
\Pi_{\mu \tau \sigma \rho}(x, y, u, v)=-\mathcal{P}_{\alpha \beta \gamma \lambda \mu \tau \sigma \rho} \frac{\partial}{\partial x_{\alpha}}\left\{\left[\frac{\partial \delta^{4}(y-x)}{\partial x_{\beta}}\right]\left[\frac{\partial \delta^{4}(u-x)}{\partial x_{\gamma}}\right]\left[\frac{\partial \delta^{4}(x-v)}{\partial x_{\lambda}}\right]\right\} .
$$

With the account of (11) and (12), the Maxwell equations (4) including the cubic nonlinearity reduce to

$$
j_{\mu}(x)=\left[\square \eta_{\mu \tau}-\partial_{\mu} \partial_{\tau}\right] a^{\tau}(x)-j_{\mu}^{\mathrm{nl}}(x),
$$

where the nonlinearly induced current (10) is reduced to

$$
j_{\mu}^{\mathrm{nl}}(x)=-\frac{1}{6} \int \mathrm{d}^{4} y \mathrm{~d}^{4} u \mathrm{~d}^{4} v \Pi_{\mu \tau \sigma \rho}(x, y, u, v) a^{\tau}(y) a^{\sigma}(u) a^{\rho}(v) .
$$

Divide the field into the sum of "linear" and "nonlinear" parts

$$
a^{\tau}(x)=a_{\operatorname{lin}}^{\tau}(x)+a_{\mathrm{nl}}^{\tau}(x)
$$

so that Eq.(17) becomes

$$
\begin{aligned}
j_{\mu}(x) & =\left[\square \eta_{\mu \tau}-\partial_{\mu} \partial_{\tau}\right] a_{\operatorname{lin}}^{\tau}(x), \\
j_{\mu}^{\text {nl }}(x) & =\left[\square \eta_{\mu \tau}-\partial_{\mu} \partial_{\tau}\right] a_{\mathrm{nl}}^{\tau}(x)
\end{aligned}
$$

In the forthcoming sections, to solve the cubic nonlinear Maxwell equations, the set (17), (18), we will be treating the nonlinearity by iterations. For a time being, however, we continue to keep it as it is, i.e. to be expressing the nonlinearly induced current $j_{\mu}^{\text {nl }}(x)$ in terms of the exact fields $a^{\tau}(x)$ as given by (18).

With the use of (16) and of integration by parts we find

$$
\begin{aligned}
& j_{\mu}^{\mathrm{nl}}(x)=\frac{\mathcal{P}_{\alpha \beta \gamma \lambda \mu \tau \sigma \rho}}{6} \frac{\partial}{\partial x_{\alpha}}\left[\int \frac{\partial \delta^{4}(y-x)}{\partial x_{\beta}} a^{\tau}(y) \mathrm{d}^{4} y \int \frac{\partial \delta^{4}(u-x)}{\partial x_{\gamma}} a^{\sigma}(u) \mathrm{d}^{4} u\right. \\
& \left.\times \int \frac{\partial \delta^{4}(x-v)}{\partial x_{\lambda}} a^{\rho}(v) \mathrm{d}^{4} v\right]=\frac{\mathcal{P}_{\alpha \beta \gamma \lambda \mu \tau \sigma \rho}}{6} \frac{\partial}{\partial x_{\alpha}}\left[\frac{\partial a^{\tau}(x)}{\partial x_{\beta}} \frac{\partial a^{\sigma}(x)}{\partial x_{\gamma}} \frac{\partial a^{\rho}(x)}{\partial x_{\lambda}}\right] .
\end{aligned}
$$

\footnotetext{
${ }^{2}$ This consequence of the correspondence principle $\mathfrak{L}_{\mathfrak{F}}=0$ is seen in the structure of the renormalized polarization operator in the momentum representation prescribed by the standard renormalization procedure, see, e.g., [16, that respects that principle, $\Pi_{\mu \tau}^{R}=$ $\left(\eta_{\mu \tau} k^{2}-k_{\mu} k_{\tau}\right)\left(\Pi\left(k^{2}\right)-\Pi(0)\right):$ this goes to zero as $\sim k^{4}$ in the infrared limit.
} 
Defining $f_{\beta \tau}(x)$ as the double of the antisymetric part of $\frac{\partial}{\partial x_{\beta}} a^{\tau}(y)$, i.e., $f^{\beta \tau}(x)=\frac{\partial}{\partial x_{\beta}} a^{\tau}(y)-\frac{\partial}{\partial x_{\tau}} a^{\beta}(y)$, and realizing that the symmetric part of $\frac{\partial}{\partial x_{\beta}} a^{\tau}(y)$ becomes zero after the contraction with $\mathcal{P}_{\alpha \beta \gamma \lambda \mu \tau \sigma \rho}$ (because it is anti-symmetric under the interchange of the respective pair of indexes: $\beta \leftrightarrow \tau, \gamma \leftrightarrow \sigma$ and $\lambda \leftrightarrow \rho$ ), we get

$$
j_{\mu}^{\mathrm{nl}}(x)=\frac{\mathcal{P}_{\alpha \beta \gamma \lambda \mu \tau \sigma \rho}}{48} \frac{\partial}{\partial x_{\alpha}}\left[f^{\beta \tau}(x) f^{\gamma \sigma}(x) f^{\lambda \rho}(x)\right] .
$$

So the nonlinearly induced current is given by

$$
j_{\mu}^{\mathrm{nl}}(x)=\frac{1}{4} \mathfrak{L}_{\mathfrak{F} \mathfrak{F}} \frac{\partial}{\partial x_{\alpha}}\left[f_{\alpha \mu}(x) f_{\beta \gamma}(x) f^{\beta \gamma}(x)\right]+\frac{1}{4} \mathfrak{L}_{\mathfrak{G} \mathfrak{G}} \frac{\partial}{\partial x_{\alpha}}\left[\tilde{f}_{\alpha \mu}(x) f_{\beta \gamma}(x) \tilde{f}^{\beta \gamma}(x)\right] .
$$

In terms of the field strengths $\mathbf{E}=\mathbf{E}(x)$ and $\mathbf{B}=\mathbf{B}(x)$, defined as $E_{i}(x)=f_{i 0}(x)$, and $B_{i}(x)=\tilde{f}_{i 0}(x)=\frac{1}{2} \epsilon_{i j k} f^{j k}(x)$, and bearing in mind that $f_{\beta \gamma}(x) f^{\beta \gamma}(x)=2\left(\mathbf{B}^{2}(x)-\mathbf{E}^{2}(x)\right)$ and $f_{\beta \gamma}(x) \tilde{f}^{\beta \gamma}(x)=-4(\mathbf{E}(x) \cdot \mathbf{B}(x))$, we obtain

$$
j_{\mu}^{\mathrm{nl}}(x)=\frac{1}{2} \mathfrak{L}_{\mathfrak{F} \mathfrak{F}} \frac{\partial}{\partial x_{\alpha}}\left[f_{\alpha \mu}(x)\left(\mathbf{B}^{2}(x)-\mathbf{E}^{2}(x)\right)\right]-\mathfrak{L}_{\mathfrak{G} \mathfrak{G}} \frac{\partial}{\partial x_{\alpha}}\left[\tilde{f}_{\alpha \mu}(x)(\mathbf{E}(x) \cdot \mathbf{B}(x))\right] .
$$

Defining $j_{\mu}^{\mathrm{nl}}(x)=\left(j_{0}^{\mathrm{nl}}(\mathbf{r}),-\mathbf{j}^{\mathrm{nl}}(\mathbf{r})\right)$, one can get the general nonlinear current in terms of the electromagnetic field

$$
\begin{aligned}
& j_{0}^{\mathrm{nl}}(x)=\frac{1}{2} \mathfrak{L}_{\mathfrak{F} \mathfrak{F}} \boldsymbol{\nabla} \cdot\left[\left(\mathbf{B}^{2}-\mathbf{E}^{2}\right) \mathbf{E}\right]-\mathfrak{L}_{\mathfrak{G} \mathfrak{G}} \boldsymbol{\nabla} \cdot[(\mathbf{E} \cdot \mathbf{B}) \mathbf{B}], \\
& \mathbf{j}^{\mathrm{nl}}(x)=-\frac{1}{2} \mathfrak{L}_{\mathfrak{F} \mathfrak{F}}\left(\frac{\partial}{\partial t}\left[\left(\mathbf{B}^{2}-\mathbf{E}^{2}\right) \mathbf{E}\right]+\boldsymbol{\nabla} \times\left[\left(\mathbf{B}^{2}-\mathbf{E}^{2}\right) \mathbf{B}\right]\right) \\
& +\mathfrak{L}_{\mathfrak{G} \mathfrak{G}}\left(\frac{\partial}{\partial t}[(\mathbf{E} \cdot \mathbf{B})] \mathbf{B}+\boldsymbol{\nabla} \times[(\mathbf{E} \cdot \mathbf{B}) \mathbf{E}]\right) .
\end{aligned}
$$

\section{Stationary nonlinear Maxwell equations in blank vacuum}

We want to solve (17), (18) imposing time independence, so we define the nonlinear electric field $\mathbf{E}^{\mathrm{nl}}$ and magnetic induction $\mathbf{B}^{\mathrm{nl}}$ as

$$
\begin{aligned}
& \mathbf{E}^{\mathrm{nl}}(\mathbf{r})=\boldsymbol{\nabla} a_{\mathrm{nl}}^{0}(\mathbf{r}), \\
& \mathbf{B}^{\mathrm{nl}}(\mathbf{r})=\boldsymbol{\nabla} \times \boldsymbol{a}_{\mathrm{nl}}(\mathbf{r}) .
\end{aligned}
$$

According to (21) these satisfy the following Maxwell equations with the stationary source $j_{\mu}^{\mathrm{nl}}(x)$

$$
\begin{aligned}
\boldsymbol{\nabla} \cdot \mathbf{E}^{\mathrm{nl}}(\mathbf{r}) & =j_{0}^{\mathrm{nl}}(\mathbf{r}), \\
\boldsymbol{\nabla} \times \mathbf{E}^{\mathrm{nl}}(\mathbf{r}) & =\mathbf{0}, \\
\boldsymbol{\nabla} \cdot \mathbf{B}^{\mathrm{nl}}(\mathbf{r}) & =0, \\
\boldsymbol{\nabla} \times \mathbf{B}^{\mathrm{nl}}(\mathbf{r}) & =\mathbf{j}^{\mathrm{nl}}(\mathbf{r}) .
\end{aligned}
$$

Note, that owing to the absence of linear response (11), field strengths and inductions are the same: $\mathbf{E}^{\mathrm{nl}}(\mathbf{r})=\mathbf{D}^{\mathrm{nl}}(\mathbf{r})$, $\mathbf{B}^{\mathrm{nl}}(\mathbf{r})=\mathbf{H}^{\mathrm{nl}}(\mathbf{r})$.

It is important to keep in mind that these Maxwell equations refer to HL units.

\subsection{Electrostatics}

To find the nonlinear electric field for a pure electrostatic problem, i.e., setting $\mathbf{B}(\mathbf{r})=0$, we start from the nonlinear charge density correction due to the fourth-rank tensor (24):

$$
j_{0}^{\mathrm{nl}}(\mathbf{r})=-\frac{1}{2} \mathfrak{L}_{\mathfrak{F} \mathfrak{F}} \nabla \cdot\left[\mathbf{E}(\mathbf{r}) E^{2}(\mathbf{r})\right],
$$

bearing in mind that the vector current density disappears in the electrostatic case, $\mathbf{j}^{\mathrm{nl}}(\mathbf{r})=0$, according to (25). Using the first equation from (27), one can write

$$
\boldsymbol{\nabla} \cdot\left[\mathbf{E}^{\mathrm{nl}}(\mathbf{r})+\frac{1}{2} \mathfrak{L}_{\mathfrak{F} \mathfrak{F}} \mathbf{E}(\mathbf{r}) E^{2}(\mathbf{r})\right]=0
$$


We introduce the notation

$$
\mathcal{E}(\mathbf{r})=-\frac{1}{2} \mathfrak{L}_{\mathfrak{F} \mathfrak{F}} \mathbf{E}(\mathbf{r}) E^{2}(\mathbf{r})
$$

The general solution to equation (29) is defined up to the curl of some vector field $\boldsymbol{\Omega}(\mathbf{r})$ :

$$
\mathbf{E}^{\mathrm{nl}}(\mathbf{r})=\mathcal{E}(\mathbf{r})+[\boldsymbol{\nabla} \times \boldsymbol{\Omega}(\mathbf{r})]
$$

The second equation from (27) allows one to fix $\Omega(\mathbf{r})$ according to

$$
[\boldsymbol{\nabla} \times[\boldsymbol{\nabla} \times \boldsymbol{\Omega}(\mathbf{r})]]=-[\boldsymbol{\nabla} \times \mathcal{E}(\mathbf{r})]
$$

in other words the vector field $\boldsymbol{\Omega}(\mathbf{r})$ must satisfy the following Poison's equation

$$
-\nabla^{2} \boldsymbol{\Omega}(\mathbf{r})+\nabla(\nabla \cdot \Omega(\mathbf{r}))=-[\boldsymbol{\nabla} \times \mathcal{E}(\mathbf{r})]
$$

The field $\boldsymbol{\Omega}(\mathbf{r})$ is defined by (32) up to a gradient: the transformation $\boldsymbol{\Omega}(\mathbf{r}) \rightarrow \boldsymbol{\Omega}^{\prime}=\boldsymbol{\Omega}+\boldsymbol{\nabla} \lambda$ leaves Eq. (32) intact. By choosing $\lambda$ to satisfy the equation $\nabla^{2} \lambda=-\boldsymbol{\nabla} \cdot \boldsymbol{\Omega}(\mathbf{r})$ we come to $\boldsymbol{\nabla} \cdot \boldsymbol{\Omega}^{\prime}=0$. Then the transformed equation (33) becomes (we omit the prime)

$$
\nabla^{2} \boldsymbol{\Omega}(\mathbf{r})=[\nabla \times \mathcal{E}(\mathbf{r})]
$$

Therefore, the solution (31) to the Maxwell equation is the longitudinal projection of the field $\mathcal{E}(\mathbf{r})(30)$, i.e.,

$$
E_{i}^{\mathrm{nl}}(\mathbf{r})=\frac{\nabla_{i} \nabla_{j}}{\nabla^{2}} \mathcal{E}_{j}(\mathbf{r})=-\frac{\nabla_{i} \nabla_{j}}{4 \pi} \int \frac{\mathcal{E}_{j}\left(\mathbf{r}^{\prime}\right)}{\left|\mathbf{r}-\mathbf{r}^{\prime}\right|} \mathrm{d} \mathbf{r}^{\prime} .
$$

or, equivalently,

$$
E_{i}^{\mathrm{nl}}(\mathbf{r})=-\frac{\nabla_{i}}{4 \pi} \int \frac{1}{\left|\mathbf{r}-\mathbf{r}^{\prime}\right|} \nabla_{j}^{\prime} \mathcal{E}_{j}\left(\mathbf{r}^{\prime}\right) \mathrm{d} \mathbf{r}^{\prime} .
$$

Note that the substitution of the Coulomb field of a point-like charge into (34) or into (35) would cause the divergency of the integral near $r^{\prime}=0$ : the present approach fails near the point charge, since it is not applicable to its strongly inhomogeneous field. Dealing with the point charge would require going beyond the infrared approximation followed to in the present work. Nevertheless, (34) (or (35)) is sound as applied to extended charges to be considered in the next section.

The nonlinear scalar potential corresponding to the field (35)

$$
a_{\mathrm{nl}}^{0}(r)=\frac{1}{4 \pi} \int \frac{\nabla^{\prime} \cdot \mathcal{E}\left(\mathbf{r}^{\prime}\right)}{\left|\mathbf{r}-\mathbf{r}^{\prime}\right|} \mathrm{d} \mathbf{r}^{\prime}
$$

\subsection{Magnetostatics}

One can calculate the nonlinear magnetic field similarly to the previous Subsection. Define

$$
\mathfrak{h}(\mathbf{r})=-\frac{1}{2} \mathfrak{L}_{\mathfrak{F} \mathfrak{F}} \mathbf{B}(\mathbf{r}) B^{2}(\mathbf{r})
$$

By setting $\mathbf{E}(\mathbf{r})=0$ in (24), (25) we find the nonlinear current for the case, where only magnetic field is developed:

$$
\begin{aligned}
j_{0}^{\mathrm{nl}}(x) & =0, \\
\mathbf{j}^{\mathrm{nl}}(x) & =\boldsymbol{\nabla} \times \mathfrak{h}(\mathbf{r}) .
\end{aligned}
$$

Then it follows from the fourth equation in (27)) that

$$
\mathbf{B}^{\mathrm{nl}}(\mathbf{r})=\mathfrak{h}(\mathbf{r})+\nabla \Phi(\mathbf{r}) .
$$

Now by the third equation from (27) one has

$$
\nabla \cdot \mathbf{B}^{\mathrm{nl}}(\mathbf{r})=\nabla \cdot \mathfrak{h}(\mathbf{r})+\nabla^{2} \Phi(\mathbf{r})=0 .
$$

Therefore, as in the electric case, the scalar field $\Phi(\mathbf{r})$ must satisfy the following Poisson equation

$$
\nabla^{2} \Phi(\mathbf{r})=-\nabla \cdot \mathfrak{h}(\mathbf{r}) .
$$


Hence, one can find the magnetic induction $\mathbf{B}^{\mathrm{nl}}(\mathbf{r})$, bearing in mind (39), as the transverse projection of the field $\mathfrak{h}(\mathbf{r})$, i.e.,

or

$$
B_{i}^{\mathrm{nl}}(\mathbf{r})=\left(\delta_{i j}-\frac{\nabla_{i} \nabla_{j}}{\nabla^{2}}\right) \mathfrak{h}_{j}(\mathbf{r})=\mathfrak{h}_{i}(\mathbf{r})+\frac{\nabla_{i} \nabla_{j}}{4 \pi} \int \frac{\mathfrak{h}_{j}\left(\mathbf{r}^{\prime}\right)}{\left|\mathbf{r}-\mathbf{r}^{\prime}\right|} \mathrm{d} \mathbf{r}^{\prime}
$$

$B_{i}^{\mathrm{nl}}(\mathbf{r})=\mathfrak{h}_{i}(\mathbf{r})+\frac{1}{4 \pi} \nabla_{i} \int \frac{\nabla_{j}^{\prime} \cdot \mathfrak{h}_{j}\left(\mathbf{r}^{\prime}\right)}{\left|\mathbf{r}-\mathbf{r}^{\prime}\right|} \mathrm{d} \mathbf{r}^{\prime}$

The corresponding vector potential is

$$
\boldsymbol{a}_{\mathrm{nl}}(\mathbf{r})=\frac{1}{4 \pi} \int \frac{\nabla^{\prime} \times \mathfrak{h}\left(\mathbf{r}^{\prime}\right)}{\left|\mathbf{r}-\mathbf{r}^{\prime}\right|} \mathrm{d} \mathbf{r}^{\prime}
$$

As far as practical applications of the equations derived in this Section for the nonlinear electric (35) and magnetic (40) fields are concerned we shall as a matter of fact take their right-hand sides with the linear approximations $\boldsymbol{E} \simeq \boldsymbol{E}^{\mathrm{lin}}(\mathbf{r})=$ $\boldsymbol{\nabla} a_{\text {lin }}^{0}(\mathbf{r}), \boldsymbol{B} \simeq \boldsymbol{B}^{\operatorname{lin}}(\mathbf{r})=\boldsymbol{\nabla} \times \boldsymbol{a}_{\text {lin }}(\mathbf{r})$ substituted into the expressions (30) for $\mathcal{E}(\boldsymbol{r})$ and (37) for $\mathfrak{h}(\mathbf{r})$, respectively. Nevertheless, certain issues can and will be discussed based on exactly nonlinear equations. For this reason we shall, for a time being, retain exact fields in (34) and (41).

\section{Applications to special static and stationary sources}

In this chapter, we are concerned about applications, like the nonlinear correction to the fields due to a charged sphere, infinite thread, infinite plane, infinite wire with a current, electric and magnetic dipoles.

\subsection{Sources providing degeneracy of the projection operators}

Equations (34), (40) obtained above map the time-independent electric and magnetic fields to their nonlinear counterparts. Generally, these mappings are nonlocal thanks to the presence of the inverse differential operator $\nabla^{-2}$. This fact also makes them more difficult to calculate. However, there are some trivial cases when a high symmetry of the problem turns the projection operators in (34) or (40) into unit operators and thereby reduces the nonlinearity to a local form. In this Subsection we concentrate on such cases, first of all on the spherically symmetric case, which is very important, because it leads to the known nonlinear correction to the Coulomb field, produced by an $\mathrm{O}(3)$-symmetrically charged sphere. If the radius of the charge is not too small (it must have low derivative, as our assumption), it may describe the electric field produced by the nuclei of some atoms. Furthermore, there are some cases, whose symmetry includes translation invariance, where charge and current distributions are not localized (infinite plane and infinite thread and wire), and it is easy to show that the projection operator is the identity operator as well.

\subsubsection{Spherical symmetry: charged sphere}

Spherical symmetry implies that any vector may be directed only along the radius-vector $\mathbf{r}$. As applied to the vector (30) this rule reads: $\mathcal{E}_{i}(\mathbf{r})=x_{i} \Lambda(r)$, where the scalar $\Lambda$ may depend only on the distance $r=\left(x_{k}^{2}\right)^{\frac{1}{2}}$. It is easy to check by direct differentiation that

$$
\nabla_{i} \nabla_{j}\left(x_{j} \Lambda(r)\right)=\nabla^{2} x_{i} \Lambda(r)
$$

i.e., the projection acts as the identity operator: $\frac{\nabla_{i} \nabla_{j}}{\nabla^{2}}=\delta_{i j}$.

Then Eq.(34) states that the corrected nonlinear electric field in the entire space is just $\mathbf{E}^{\mathrm{nl}}(\mathbf{r})=\mathcal{E}(\mathbf{r})=-\frac{1}{2} \mathfrak{L}_{\mathfrak{F} \mathfrak{F}} E^{2}(r) \mathbf{E}(\mathbf{r})$. Hence, within the cubic approximation (4) the total (nonlinearly corrected) electric field, equal to $\mathbf{E}(\mathbf{r})=\mathbf{E}^{\text {lin }}(\mathbf{r})+\mathbf{E}^{\mathrm{nl}}(\mathbf{r})$ in accord with (19), is subject to the following cubic equation

$$
\mathbf{E}(\mathbf{r})=\mathbf{E}^{\operatorname{lin}}(\mathbf{r})-\frac{1}{2} \mathfrak{L}_{\mathfrak{F} \mathfrak{F}} \mathbf{E}(\mathbf{r}) E^{2}(r) .
$$

With the Euler-Heisenberg Lagrangian [17] one can calculate that $\mathfrak{L}_{\mathfrak{F} \mathfrak{F}}=\frac{e^{4}}{45 \pi^{2} m^{4}}$, where $m$ is the electron mass, while its charge $e$ is to be taken in the same system of units as $\mathfrak{L}_{\mathfrak{F} \mathfrak{F}}$.

As long as this coefficient is very small it hardly makes sense to treat the nonlinearity in equation (44) seriously. Instead, it is sufficient to keep $\mathbf{E}(\mathbf{r}) \simeq \mathbf{E}^{\operatorname{lin}}(\mathbf{r})$ in its right-hand side. Then the approximate solution to this equation is given as

$$
\mathbf{E}(\mathbf{r})=\mathbf{E}^{\operatorname{lin}}(\mathbf{r})\left(1-\frac{2 \alpha}{45 \pi}\left(\frac{e E^{\operatorname{lin}}(r)}{m^{2}}\right)^{2}\right) .
$$


This expression can be trusted for the field values $E^{\operatorname{lin}}(\mathbf{r}) \lesssim \frac{m^{2}}{e} \sqrt{\frac{45 \pi}{2 \alpha}}$. This upper bound of applicability is higher than Schwinger's characteristic value $E_{\mathrm{Sch}}=\frac{m^{2}}{e}$. However, if the field compares with this characteristic value and exceeds it, the vacuum instability via spontaneous production of electron-positron pairs [21] is expected to become more and more essential, which fact would require a revision of our result.

If the standard Coulomb field $\frac{q}{4 \pi r^{2}} \frac{\mathbf{r}}{r}$ linearly produced by a point charge $q$ is used for $\mathbf{E}^{\text {lin }}(\mathbf{r})$ in (45) the latter is reduced to an expression for the nonlinear correction to this field in the vacuum found in [17], which is highly singular in the origin $r=0$. For the atomic field with $q=Z e$ and $Z$ about a few tens, the essential correction of $10^{-5}$ is achieved at the distance from the nucleus about the electron Compton length.

Equation (45) can be applied to an extended spherically-symmetric charge $q$, say, a homogeneously charged sphere with the radius $R$. We define two regions $r<R$ and $r>R$, and the linear electric field is given by

$$
\mathbf{E}^{\operatorname{lin}}(r)=\Theta(R-r) \mathbf{E}^{<}(r)+\Theta(r-R) \mathbf{E}^{>}(r)
$$

where

$$
\mathbf{E}^{<}(r)=\frac{q r}{4 \pi R^{3}} \mathbf{e}_{r}, \quad \mathbf{E}^{>}(r)=\frac{q}{4 \pi r^{2}} \mathbf{e}_{r} .
$$

This field should be used in (45) to produce a finite value in the origin $r=0$.

\subsubsection{Cylindric plus translation symmetry: charged plane, charged thread, and current-carrying wire}

Three other cases, when the projections in (34) or in (40) reduce to trivial identities may be revealed.

One of them is supplied by the translation invariance along every plane orthogonal to a given axis (axis 3 , for instance), characteristic of a finite-thickness infinite plane charged homogeneously along the directions 1 and 2 . Then $\mathcal{E}_{1,2}(\mathbf{r})=0$, $\mathcal{E}_{3}(\mathbf{r})=x_{3} \Lambda\left(x_{3}\right)$, hence, when applied to $\mathcal{E}(\mathbf{r})$, the projection operator is unity: $\frac{\nabla_{i} \nabla_{j}}{\nabla^{2}}=\delta_{i j}$. Then, in place of $(45)$ we get

$$
E_{3}\left(x_{3}\right)=E_{3}^{\operatorname{lin}}\left(x_{3}\right)\left(1-\frac{2 \alpha}{45 \pi}\left(\frac{e E^{\operatorname{lin}}\left(x_{3}\right)}{m^{2}}\right)^{2}\right), \quad E_{1,2}=0 .
$$

If, besides, the volume charge density $\kappa$ is also homogeneous along the axis 3 , so that finally $\kappa=$ const, and the plane is cituated symmetrically with respect to the coordinate plane $x_{3}=0$, its standard electric field

$$
\begin{gathered}
\mathbf{E}^{\operatorname{lin}}\left(x_{3}\right)=\Theta\left(\frac{d}{2}-\left|x_{3}\right|\right) \mathbf{E}^{<}\left(x_{3}\right)+\Theta\left(\left|x_{3}\right|-\frac{d}{2}\right) \mathbf{E}^{>}\left(x_{3}\right), \\
\mathbf{E}^{<}\left(x_{3}\right)=\kappa x_{3} \mathbf{e}_{3}, \quad \mathbf{E}^{>}\left(x_{3}\right)=\frac{\kappa d}{2} \operatorname{sgn}\left(x_{3}\right) \mathbf{e}_{3},
\end{gathered}
$$

where $\operatorname{sgn}\left(x_{3}\right)=\frac{\left|x_{3}\right|}{x_{3}}$ and $d$ is the thickness of the plane, should be used in (48) for obtaining the nonlinear extension of the field produced by this charged plane.

Another case of trivialization of the projection operator is provided by the cylindric symmetry under rotations around the axis 3 supplemented by the invariance under translations along this axis. This type of symmetry is peculiar to an infinite round-cylindric thread homogeneously charged along itself and $\mathrm{O}(2)$-symmetrically charged across. Then $\mathcal{E}_{3}(\mathbf{r})=0, \mathcal{E}_{1,2}(\mathbf{r})=\rho_{1,2} \Lambda(\rho)$, where $\boldsymbol{\rho}$ is the radius-vector in the (1,2)-plane. In this case, it is again easy to check by direct differentiation that $\boldsymbol{\nabla}(\boldsymbol{\nabla} \cdot \boldsymbol{\rho} \Lambda(\rho))=\nabla^{2} \boldsymbol{\rho} \Lambda(\rho)$ and hence the projection operator in (34) is unity. Then, in place of (45) the relation

$$
E_{1,2}(\rho)=E_{1,2}^{\operatorname{lin}}(\rho)\left(1-\frac{2 \alpha}{45 \pi}\left(\frac{e\left|\mathbf{E}^{\operatorname{lin}}(\rho)\right|}{m^{2}}\right)^{2}\right), \quad E_{3}=0
$$

should hold. If, besides, the volume charge density $\kappa$ is independent also on the radius $\rho, \kappa=$ const, the standard electric field of the homogeneously charged straight thread with the radius $R$ and its axis coinciding with the coordinate axis 3

$$
\begin{gathered}
\mathbf{E}^{\operatorname{lin}}(\rho)=\Theta(R-\rho) \mathbf{E}^{<}(\rho)+\Theta(\rho-R) \mathbf{E}^{>}(\rho), \\
\mathbf{E}^{<}(\rho)=\frac{\kappa \rho}{2} \mathbf{e}_{\rho}, \mathbf{E}^{>}(\rho)=\frac{\kappa R^{2}}{2 \rho} \mathbf{e}_{\rho}, \mathbf{e}_{\rho}=\frac{\boldsymbol{\rho}}{\rho}
\end{gathered}
$$

is to be substituted into (51). 
The same symmetry as that of the infinite thread above is inherent to the straight infinite round-cylindric wire carrying a

constant current with $\mathrm{O}(2)$-invariant density. This time, the field (37) has the structure $\mathfrak{h}(\mathbf{r})=\boldsymbol{e}_{3} \times \mathbf{r} \Lambda\left(\left|\boldsymbol{e}_{3} \times \mathbf{r}\right|\right)$, where $\boldsymbol{e}_{3}$ is the unit vector along axis 3 , and $\left|\boldsymbol{e}_{3} \times \mathbf{r}\right|=\rho$. Now $\boldsymbol{\nabla} \cdot \mathfrak{h}(\mathbf{r})=0$, and the projection operator in (40) is unity again, so that $B_{i}^{\text {nl }}(\mathbf{r})=\mathfrak{h}_{i}(\mathbf{r})$.

Now if we take a fully homogeneous current density $\mathbf{j}$ per unit area, flowing along the positive direction, and calculate the linear magnetic field produced by it, we get the standard relation

$$
\begin{aligned}
& \mathbf{B}^{\operatorname{lin}}(\rho)=\Theta(R-\rho) \mathbf{B}^{<}(\rho)+\Theta(\rho-R) \mathbf{B}^{>}(\rho), \\
& \mathbf{B}^{<}(\rho)=\frac{j \rho}{2} \mathbf{e}_{\phi}, \mathbf{B}^{>}(\rho)=\frac{j R^{2}}{2 \rho} \mathbf{e}_{\phi}, \mathbf{e}_{\phi}=\frac{\mathbf{e}_{3} \times \boldsymbol{\rho}}{\rho}
\end{aligned}
$$

to be used in the expression

$$
B_{\phi}(\rho)=B_{\phi}^{\operatorname{lin}}(\rho)\left(1-\frac{2 \alpha}{45 \pi}\left(\frac{e B_{\phi}^{\operatorname{lin}}(\rho)}{m^{2}}\right)^{2}\right)
$$

that follows from (40) and (37) for the present type of symmetry of the problem.

\subsection{Cylindric symmetry: Elementary dipoles}

In this Subsection, we proceed with other cases of cylindric symmetry, but without any translation invariance. We will calculate nonlinear corrections to the fields of magnetic and electric dipoles by realizing nontrivial projections in (34) or in (40).

\subsubsection{Magnetic dipole.}

Let there be a sphere with the radius $R$, and a time-independent current $\mathbf{j}(\mathbf{r})$ concentrated on its surface:

$$
\mathbf{j}(\mathbf{r})=3 \frac{\mathcal{M} \times \mathbf{r}}{r^{4}} \delta(r-R) .
$$

Here $\mathcal{M}$ is a constant vector directed, say, along the axis 3 . The current density (57) obeys the continuity condition $\nabla \cdot \mathbf{j}(\mathbf{r})=0$, its flow lines are circular in the planes parallel to the plane $(1,2)$. The magnetic field produced by this current via the Maxwell equation $\nabla \times \boldsymbol{B}^{\operatorname{lin}}(\mathbf{r})=\mathbf{j}(\mathbf{r})$ is, everywhere outside of the sphere, the magnetic dipole field 3

$$
\mathbf{B}^{>}=-\frac{\mathcal{M}}{r^{3}}+3 \frac{\mathbf{r} \cdot \mathcal{M}}{r^{5}} \mathbf{r},
$$

with the constant vector density $\mathcal{M}$ introduced in (57) playing the role of the corresponding magnetic moment. Inside the sphere the magnetic field is constant:

$$
\mathbf{B}^{<}=\frac{2 \mathcal{M}}{R^{3}}
$$

It turns to infinity for a point-like dipole $R=0$.

We should stress that Eqs. (58), (59) are used to difine the magnetic moment both in the (rationalized) HeavisideLorentz and Gauss systems of units, provided that the right- and left-hand sides refer to one and the same system. This form of equation may be called dimension-covariant.

We have

$$
\mathbf{B}^{\operatorname{lin}}(\mathbf{r})=\Theta(R-r) \mathbf{B}^{<}(\mathbf{r})+\Theta(r-R) \mathbf{B}^{>}(\mathbf{r})
$$

where $\Theta(x)=\left\{\begin{array}{cc}1, & \text { if } x>0, \\ 0, & \text { if } x<0 .\end{array}\right.$ is the step function. Each function $\mathbf{B} \gtrless(\mathbf{r})$ satisfies the sourceless Maxwell equation $\nabla \times \boldsymbol{B}^{\gtrless}(\boldsymbol{r})=0$, whereas the delta function in the current density (57) is produced by differentiation of the step function in (60) under the curl operation. The relative coefficient 2 in (59) is chosen so as to make the field also satisfy the other Maxwell equation $\nabla \cdot \boldsymbol{B}^{\text {lin }}(\boldsymbol{r})=0$. Consequently, the radial component $\boldsymbol{r} \cdot \mathbf{B}^{\text {lin }}(\mathbf{r})$ of the field (60) is continuous at the

\footnotetext{
${ }^{3}$ The extra factor of $4 \pi$ should appear in (57) in the Gauss system in accordance with the fact that this factor is present in the right-hand side of the Maxwell equation used.
} 
border of the sphere $r=R$, while its tangent component in the plane spanned by the vectors $\boldsymbol{r}$ and $\mathcal{M}$, is not. (Its component in the plane orthogonal to $\boldsymbol{r}$ and $\mathcal{M}$ disappears). We write the field (60) as

$$
\begin{aligned}
& \mathbf{B}^{\operatorname{lin}}(\mathbf{r})=\left(\frac{2 \mathcal{M}}{R^{3}} \cos \theta \mathbf{e}_{r}-\frac{2 \mathcal{M}}{R^{3}} \sin \theta \mathbf{e}_{\theta}\right) \Theta(R-r) \\
& +\left(\frac{2 \mathcal{M}}{r^{3}} \cos \theta \mathbf{e}_{r}+\frac{\mathcal{M}}{r^{3}} \sin \theta \mathbf{e}_{\theta}\right) \Theta(r-R),
\end{aligned}
$$

where $\theta$ is the angle between the vectors $\boldsymbol{r}$ and $\mathcal{M}$, the unit vectors $\mathbf{e}_{r}$ and $\mathbf{e}_{\theta}$ are, respectively, along $\boldsymbol{r}$ and along the tangent direction in the plane spanned by these vectors, directed in such a way that $\mathbf{e}_{\theta}$ be opposite to $\mathcal{M}$ at $\theta=\pi / 2$. Let us calculate the field (37) taken on the linear expression (61) for $\boldsymbol{B}$

$$
\begin{aligned}
& -\frac{2 \mathfrak{h}(\mathbf{r}) R^{9}}{\mathfrak{L}_{\mathfrak{F} \mathfrak{F}} \mathcal{M}^{3}}=\left(8 \cos \theta \mathbf{e}_{r}-8 \sin \theta \mathbf{e}_{\theta}\right) \Theta(R-r) \\
& +\left[\left(2 \frac{R^{9}}{r^{9}} \cos \theta+6 \frac{R^{9}}{r^{9}} \cos ^{3} \theta\right) \mathbf{e}_{r}+\left(4 \frac{R^{9}}{r^{9}} \sin \theta-3 \frac{R^{9}}{r^{9}} \sin ^{3} \theta\right) \mathbf{e}_{\theta}\right] \Theta(r-R) .
\end{aligned}
$$

The further calculations of the nonlinear magnetic field of a dipole following (41) are traced in Appendix 2. The result is

$$
\begin{aligned}
& \frac{-2 R^{9}}{\mathfrak{L}_{\mathfrak{F} \mathfrak{F}} \mathcal{M}^{3}} \mathbf{B}^{\mathrm{nl}}(\mathbf{r})= \\
& \left\{\left[\left(\frac{88}{15}-\frac{324}{385} \frac{r^{2}}{R^{2}}\right) \cos \theta+\frac{108}{77} \frac{r^{2}}{R^{2}} \cos ^{3} \theta\right] \mathbf{e}_{r}+\left[\left(-\frac{88}{15}-\frac{432}{385} \frac{r^{2}}{R^{2}}\right) \sin \theta+\frac{108}{77} \frac{r^{2}}{R^{2}} \sin ^{3} \theta\right] \mathbf{e}_{\theta}\right\} \Theta(R-r) \\
& +\left\{\left[\left(\frac{28}{5} \frac{R^{3}}{r^{3}}-\frac{18}{35} \frac{R^{5}}{r^{5}}-\frac{2}{33} \frac{R^{9}}{r^{9}}\right) \cos \theta+\left(\frac{6}{7} \frac{R^{5}}{r^{5}}+\frac{6}{11} \frac{R^{9}}{r^{9}}\right) \cos ^{3} \theta\right] \mathbf{e}_{r}\right. \\
& \left.+\left[\left(\frac{14}{5} \frac{R^{3}}{r^{3}}+\frac{18}{35} \frac{R^{5}}{r^{5}}+\frac{56}{33} \frac{R^{9}}{r^{9}}\right) \sin \theta+\left(-\frac{9}{14} \frac{R^{5}}{r^{5}}-\frac{21}{22} \frac{R^{9}}{r^{9}}\right) \sin ^{3} \theta\right] \mathbf{e}_{\theta}\right\} \Theta(r-R) .
\end{aligned}
$$

For the inside region of the sphere, i.e., when $r<R$, we see that the solution is regular at the origin and directed, as expected, along $\mathcal{M}: \mathbf{B}^{\text {nl }}(0)=-\frac{44}{15 R^{9}} \mathfrak{L}_{\mathfrak{F} \mathfrak{F}} \mathcal{M}^{3} \mathbf{e}_{3}$. For the outside region of the sphere, i.e., when $r>R$, we see that the field has three powers of the ratio of the distance from the origin to the radius of the sphere: the 9th, the 5th and, most interesting, the 3rd power, the latter just like the linear field (60). This means that, at large distances from the origin, the correction to the field behaves in the same way as the linear field. (This is in contrast to the field of electric monopole given by (45) after the Coulimb field is substituted into it, where the correction decreases as the 6th power, while the linear (Coulomb) field decreases as the 2nd power.) Therefore, imposing $r>>R$

$$
\left.\mathbf{B}^{\mathrm{nl}}(\mathbf{r})\right|_{r>>R}=-\frac{7}{5} \mathfrak{L}_{\mathfrak{F} \mathfrak{F}} \frac{\mathcal{M}^{3}}{R^{6} r^{3}}\left(2 \cos \theta \mathbf{e}_{r}+\sin \theta \mathbf{e}_{\theta}\right) .
$$

In terms of the linear field (60) the total (nonlinearly corrected) field is the sum of the linear field and the nonlinear field, according to (19)

$$
\left.\mathbf{B}(\mathbf{r})\right|_{r>>R}=\mathbf{B}^{\operatorname{lin}}(\mathbf{r})\left(1-\frac{7}{5} \mathfrak{L}_{\mathfrak{F} \mathfrak{F}} \frac{\mathcal{M}^{2}}{R^{6}}\right) .
$$

Note that unlike Eq. (61), the expressions (63), (64), (65) refer only to HL system, since Eq. (41) is a solution to the Maxwell equation (27) written in that system.

For the Euler-Heisenberg Lagrangian one has

$$
\mathfrak{L}_{\mathfrak{F} \mathfrak{F}}=\frac{e^{4}}{45 \pi^{2} m^{4}}=\frac{e^{2}}{45 \pi^{2}}\left(\frac{1}{B_{\mathrm{Sch}}}\right)^{2} .
$$

Once the Euler-Heisenberg Lagrangian $\mathfrak{L}$ is a function of the product $e B$ (or of $e^{2} \mathfrak{F}$ ), it is dimension-invariant, i.e. the same in HL and Gauss systems. Hence the variational derivative $\mathfrak{L}_{\mathfrak{F} \mathfrak{F}}(66)$ is dimension-covariant. This implies that in it one may take $e$ either equal to its Gauss value $e^{\mathrm{G}}=(1 / 137)^{1 / 2}$ and, correspondingly, choose $B_{\text {Sch }}=B_{\text {Sch }}^{\mathrm{G}}=m^{2} / e^{\mathrm{G}}=4.4 \cdot 10^{13} \mathrm{G}$, or, alternately, take $e=e^{\mathrm{HL}}=(4 \pi / 137)$ and $B_{\mathrm{Sch}}=B_{\mathrm{Sch}}^{\mathrm{HL}}=m^{2} / e^{\mathrm{Hl}}=1.24 \cdot 10^{13} \mathrm{G}$. It is understood that in the two cases the field squared $\mathfrak{F}$ inside $\mathfrak{L}_{\mathfrak{F} \mathfrak{F}}$ should be simultaneously normalized differently. However, when substituting (66) in (65) only HL values should be used. So, in QED the overall field of the magnetic dipole is given by

$$
\begin{aligned}
& \left.\mathbf{B}(\mathbf{r})\right|_{r>>R}=\mathbf{B}^{\operatorname{lin}}(\mathbf{r})\left(1-\frac{7}{5} \frac{e^{2}}{45 \pi^{2}}\left(\frac{e}{m^{2}} \frac{\mathcal{M}}{R^{3}}\right)^{2}\right) \\
& =\mathbf{B}^{\operatorname{lin}}(\mathbf{r})\left(1-\frac{7}{5} \frac{e^{2}}{180 \pi^{2}}\left(\frac{B^{<}}{B^{\text {Sch }}}\right)^{2}\right) .
\end{aligned}
$$


The last equation is to be presented in the final form, independent of a system of units

$$
\left.\mathbf{B}(\mathbf{r})\right|_{r>>R}=\mathbf{B}^{\operatorname{lin}}(\mathbf{r})\left(1-\frac{7}{5} \frac{\alpha}{45 \pi}\left(\frac{B^{<}}{B^{\mathrm{Sch}}}\right)^{2}\right) \text {. }
$$

Once the form (64) - 68 ) of the magnetic dipole field far from the source proved to be invariant under nonlinear correction, we may abandon the approximation $\mathbf{B} \simeq \mathbf{B}^{\operatorname{lin}}(\mathbf{r})$ used when calculating $\mathfrak{h}(\mathbf{r})$ above. This means that $\mathcal{M}$ in (62), (63) and (64) may be thought of as the final, nonlinearly corrected, magnetic moment $\mathcal{M}^{\text {nlc }}$. Then it becomes subject to the cubic equation

$$
\mathcal{M}^{\text {nlc }}=\mathcal{M}-\frac{7}{5} \mathfrak{L}_{\mathfrak{F} \mathfrak{F}} \mathcal{M}^{\text {nlc }}\left(\frac{\mathcal{M}^{\mathrm{nlc}}}{R^{3}}\right)^{2} .
$$

This equation shows nonlinear self-coupling of the magnetic moment. The minus sign here excludes its spontaneous production when the "bare" magnetic moment is not present $(\mathcal{M}=0)$ as long as $\mathfrak{L}_{\mathfrak{F} \mathfrak{F}}>0$. (The positivity of $\mathfrak{L}_{\mathfrak{F} \mathfrak{F}}$ is a consequence of causality and unitarity [22]). In the approximation of $\mathbf{B} \simeq \mathbf{B}^{\operatorname{lin}}(\mathbf{r})$ the correction to the magnetic moment looks as

$$
\mathcal{M}^{\mathrm{nlc}}=\mathcal{M}\left(1-\frac{7}{5} \mathfrak{L}_{\mathfrak{F} \mathfrak{F}} \frac{\mathcal{M}^{2}}{R^{6}}\right) .
$$

\subsubsection{Electric dipole.}

Let there be a sphere with the radius $R$, and a time-independent charge $j_{0}(\mathbf{r})$ concentrated on its surface:

$$
j_{0}(\mathbf{r})=3 \frac{\mathbf{p} \cdot \mathbf{r}}{r^{4}} \delta(r-R)
$$

Here $\mathbf{p}$ is a constant vector directed, say, "to the North", i.e. along the axis 3. The current density (71) obeys the continuity condition $\partial_{0} j_{0}=0$. It is continuously distributed over the surface, the "northern" hemisphere being positevely, and "southern" hemisphere negatively charged. The extremum charge densities are achieved at the poles, while the equator remains neutral. The electric field produced by this current via the Maxwell equation $\nabla \cdot \mathbf{E}^{\text {lin }}(\mathbf{r})=j_{0}(\mathbf{r})$ is, outside of the sphere $r>R$, where $j_{0}(\mathbf{r})=0$, the electric dipole field

$$
\mathbf{E}^{>}(\mathbf{r})=-\frac{\mathbf{p}}{r^{3}}+3 \frac{\mathbf{r} \cdot \mathbf{p}}{r^{5}} \mathbf{r}
$$

with the constant vector density p playing the role of the corresponding electric moment. Inside the sphere the electric field is constant:

$$
\mathbf{E}^{<}(\mathbf{r})=-\frac{\mathbf{p}}{R^{3}}
$$

It turns to infinity for a point-like dipole $R=0$. We have

$$
\mathbf{E}^{\text {lin }}(\mathbf{r})=\Theta(R-r) \mathbf{E}^{<}(\mathbf{r})+\Theta(r-R) \mathbf{E}^{>}(\mathbf{r}) .
$$

Each function $\mathbf{E} \lessgtr(\mathbf{r})$ satisfies the sourceless Maxwell equation $\nabla \cdot \mathbf{E} \lessgtr(\mathbf{r})=0$, whereas the delta function in the current density (71) is produced by differentiation of the step function $\Theta$ in (74) under the divergence operation in the Maxwell equation $\nabla \cdot \mathbf{E}^{\text {lin }}(\mathbf{r})=j_{0}(\mathbf{r})$. The relative coefficient -1 in (73) is chosen so as to make the field also satisfy the other

Maxwell equation $\nabla \times \mathbf{E}^{\text {lin }}(\mathbf{r})=0$. Consequently, the component $\mathbf{e}_{\theta} \cdot \mathbf{E}^{\text {lin }}(\mathbf{r})$ of the field (74) tangent to the sphere is continuous at the border of the sphere $r=R$, while its perpendicular component $\mathbf{r} \cdot \mathbf{E}^{\text {lin }}(\mathbf{r})$ is not.

By doing the same calculations as in the previous Subsection (see Appendix 3) we find the nonlinear correction to the electric field of the dipole taken in the approximation $\mathbf{E}(\mathbf{r}) \simeq \mathbf{E}^{\operatorname{lin}}(\mathbf{r})$ (now $\theta$ is the angle between $\mathbf{r}$ and $\mathbf{p}$ ) :

$$
\begin{aligned}
& \frac{-2 R^{9}}{\mathfrak{L}_{\mathfrak{F} \mathfrak{F}} p^{3}} \mathbf{E}^{\mathrm{nl}}(\mathbf{r})= \\
& \left\{\left[\left(-\frac{13}{15}+\frac{324}{385} \frac{r^{2}}{R^{2}}\right) \cos \theta-\frac{108}{77} \frac{r^{2}}{R^{2}} \cos ^{3} \theta\right] \mathbf{e}_{r}\right. \\
& \left.+\left[\left(\frac{13}{15}+\frac{432}{385} \frac{r^{2}}{R^{2}}\right) \sin \theta-\frac{108}{77} \frac{r^{2}}{R^{2}} \sin ^{3} \theta\right] \mathbf{e}_{\theta}\right\} \Theta(R-r) \\
& +\left\{\left[\left(\frac{2}{5} \frac{R^{3}}{r^{3}}+\frac{18}{35} \frac{R^{5}}{r^{5}}+\frac{68}{33} \frac{R^{9}}{r^{9}}\right) \cos \theta+\left(-\frac{6}{7} \frac{R^{5}}{r^{5}}+\frac{60}{11} \frac{R^{9}}{r^{9}}\right) \cos ^{3} \theta\right] \mathbf{e}_{r}\right. \\
& \left.+\left[\left(\frac{1}{5} \frac{R^{3}}{r^{3}}-\frac{18}{35} \frac{R^{5}}{r^{5}}+\frac{76}{33} \frac{R^{9}}{r^{9}}\right) \sin \theta+\left(\frac{9}{14} \frac{R^{5}}{r^{5}}-\frac{45}{22} \frac{R^{9}}{r^{9}}\right) \sin ^{3} \theta\right] \mathbf{e}_{\theta}\right\} \Theta(r-R)
\end{aligned}
$$


At large distances we have

$$
\left.\mathbf{E}(\mathbf{r})\right|_{r>>R}=\mathbf{E}^{\operatorname{lin}}(\mathbf{r})+\left.\mathbf{E}^{\mathrm{nl}}(\mathbf{r})\right|_{r>>R}=\mathbf{E}^{\operatorname{lin}}(\mathbf{r})\left(1-\frac{1}{10} \mathfrak{L}_{\mathfrak{F} \mathfrak{F}}\left(\frac{p}{R^{3}}\right)^{2}\right),
$$

where $\mathbf{E}^{\text {lin }}(\mathbf{r})=\mathbf{E}^{>}(\mathbf{r})=\frac{p}{r^{3}}\left(2 \cos \theta \mathbf{e}_{r}+\sin \theta \mathbf{e}_{\theta}\right)$ is the (outside) linear electric field (72). With the Euler-Heisenberg Lagrangian we use (66) for $\mathfrak{L}_{\mathfrak{F} \mathfrak{F}}$. Then the overall field in QED is given by

$$
\left.\mathbf{E}(\mathbf{r})\right|_{r>>R}=\mathbf{E}^{\operatorname{lin}}(\mathbf{r})\left(1-\frac{2}{45} \frac{\alpha}{45 \pi}\left(\frac{E^{<}}{E^{\mathrm{Sch}}}\right)^{2}\right),
$$

where $E^{\mathrm{Sch}}$ is Schwinger's characteristic value for electric field. The form (77) is valid both in HL and Gauss systems of units.

The statements, made about the magnetic moment $\mathcal{M}$ and its magnetic field at the end of the previous Subsection, can be repeated as applied to the electric moment $\mathbf{p}$ and its electric field. For instance, the analog of (69) is the equation for selfinteracting electric dipole model

$$
\mathbf{p}^{\mathrm{nlc}}=\mathbf{p}-\frac{\mathbf{p}^{\mathrm{nlc}}}{10} \mathfrak{L}_{\mathfrak{F} \mathfrak{F}}\left(\frac{p^{\mathrm{nlc}}}{R^{3}}\right)^{2}
$$

where $\mathbf{p}$ is the "bare" moment, introduced in (71), and $\mathbf{p}^{\text {nlc }}$ is the nonlinerly corrected moment.

The vast difference of the results (76) - (77) of the present Subsection from those relating to the spherical-symmetric case of Subsection 4.1.1, namely, from Eqs. (44), (45), is that the correction in (76) - (77) contains only internal properties of the source, and does not depend on the distance from it, in contrast to (44), (45). Eqs. (76) - (77) may be thought of as a sort of renormalization of the dipole moment $p$, whereas (45) is not a correction to the charge $q$, but rather the field renormalization. The same remark is valid also for the magnetic dipole of the previous Subsection, Eqs. (64) - (70).

\section{Some numerical estimates}

The nonlinear corrections to electromagnetic fields in the blank vacuum considered in the previous sections are cubic with respect to the primary, linear fields. This means that these are most essential for large fields. In this section we discuss numerically a few important instances of strong fields.

\subsection{Baryons}

The of the neutron magnetic moment in nuclear magnetons $\mu_{N}=\frac{e}{2 m_{\mathrm{p}}}$, where $m_{\mathrm{p}}$ is the proton mass (remind that, in Gauss units, $\left.\frac{e \hbar}{2 m_{\mathrm{p}} c}=5.05078324 \cdot 10^{-24} \mathrm{erg} / \mathrm{G}\right)$ is measured [23] with the high precision of $\sigma_{\mathcal{M}}=0.0000005 \mu_{N}$ to be

$$
\mathcal{M}=-1.9130427 \mu_{N}
$$

The magnetic radius of the neutron is believed to make (up to the third decimal point)

$$
R=0.862 \mathrm{fm} .
$$

Linear magnetic field (58) of the neutron due to its magnetic moment at a distance $r$ (along the magnetic moment direction) is $B^{\text {lin }}=2 \frac{\mathcal{M}}{r^{3}}$. It reaches Schwinger's critical value, equal to $B_{\mathrm{Sch}}=4.4 \times 10^{13} \mathrm{G}$ in Gauss units, at $r=r_{\mathrm{c}}=7.55$ fm.

Then, the field exceeds Schwinger's value, when we are closer to the neutron than its $7.55 / 0.86=8.8$ radii.

The magnetization of a neutron, defined within the simple model of Subsection 4.2.1 as $M=\frac{3 \mathcal{M}}{4 \pi R^{3}}$ makes in Gauss units

$$
M=3.75 \times 10^{15} \mathrm{G}=852 B_{\mathrm{Sch}},
$$

and the magnetic field (59) at its electromagnetic radius (80) is about $3 \cdot 10^{16} \mathrm{G}$. The value (81) is of the order of magnetization of a strongest magnetar field and may be understood as magnetization of a neutron star, provided the latter is viewed upon as a spontaneously magnetized dense matter of paralelly arranged magnetic dipoles, neutrons, if they are as close to each other as their magnetic radius (80). (The corresponding density of matter would be $6.2 \cdot 10^{14}$ $\left.\mathrm{g} / \mathrm{cm}^{3}\right)$. For the proton the value of magnetization is twice as large, $7.5 \times 10^{16} \mathrm{G}=1.7 \cdot 10^{3} B_{\mathrm{Sch}}$. 
With so large magnetization, the nonlinear correction in (70), calculated with the use of (66)

$$
\frac{7}{5} \mathfrak{L}_{\mathfrak{F} \mathfrak{F}}\left(\frac{\mathcal{M}}{R^{3}}\right)^{2}=\frac{28}{225 \pi} \alpha\left(\frac{M}{B_{\mathrm{Sch}}}\right)^{2}=2.89\left(\frac{1.9 \cdot 5.05}{4.4(0.86)^{3}}\right)^{2}=33.5>>1,
$$

takes us far out of the scope of validity of the power expansion (44). Therefore, for treating the neutron and proton, as well as the most of other baryons, we must find a way of going beyond this framework. While leaving this task for future work, we, however, can indicate now that the results of Subsubsection 4.2.1 are ready for service when particles or resonances with smaller magnetic moments are concerned. We attract attention to the excited baryon $\Xi^{* 0}$, whose magnetic moment is estimated [24] at the level of 0.16(4), and to the $\Delta^{0}$ decuplet member, with the magnetic moment $-0.035(2)$. Then the nonlinear correction to magnetization (and to its magnetic moment as well, assuming the magnetic radius being of the same order as that of the neutron) makes $0.23<<1$ for $\Xi^{* 0}$ and $0.01<<1$ for $\Delta^{0}$. The correction for $\Xi^{* 0}$ is within the range of admitted theoretical errors in Ref. 24, that reports the calculations of magnetic moments appealing to the quark structure and fulfilled within the lattice QCD with the extrapolation to the observable value of the pion mass (with the help of the chiral perturbation theory). As for $\Delta^{0}$, the above nonlinear correction 0.01 somewhat falls off of the admitted range of $2 / 35=0.057$. But other calculations (those of Ref. 25] made with the help of chiral perturbation theory) give $-0.17(4)$ for the magnetic moment of $\Delta^{0}$, and, correspondingly, produce the nonlinear correction of 0.26 , while the theoretical indeterminacy is 0.23 . Another candidate treatable with our formulas may be $\Sigma^{* 0}$. Experimental values do not exist for these baryons, but there are theoretical predictions within the same theory that produces good coincidence with experimentally measured magnetic moments wherever the latter are available, neutron and proton included.

We conclude that the nonlinear correction to the magnetic moment of the above baryons, as lying within the range of existing theoretical indeterminacy and, perhaps, within its discrepancy with a future experiment, indicates that it may come seriously into play already at the very next step of improving the theoretical results.

\subsection{Pulsars}

Near the surface at the north magnetic pole of a fast rotating radio-pulsar [18] (also for certain magnetars [26]) we may

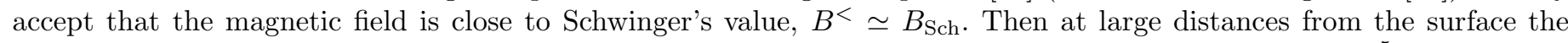
relative nonlinear correction to the magnetic field (the second term in the brace of Eq. (68) ) is $\simeq 7 \cdot 10^{-5}$, the absolute correction to the field being thus $\simeq 3 \cdot 10^{9} \mathrm{G}$. Some magnetars [27] (soft gamma-ray repeaters, anomaleous X-ray pulsars etc.) are believed to have the magnetic fields up to $10^{15} \mathrm{G}$, (and, under certain assumptions, even two orders larger [28]). Then, in absolute value the nonlinear correction may achieve the huge value of somewhat below $10^{14} \mathrm{G}$, which may influence the fundamental physical processes in the neutron star magnetosphere and hence the mechanisms of their radiation.

We can rely on Eqs. (64) - (70) till the correction in them does not exceed, say, 10\%. For this case Fig. 1, drawn following Eq. (63) and corresponding to the field at the surface equal to $B^{<}=37 B_{\text {Sch }}$, shows how the ratio between the nonlinear field and the linear field behaves as the distance to the surface increases. Whereas at large distances from the pulsar the corrected field follows the same magnetic dipole law (58) as the primary, linear field, near the surface it considerably deviates from the field of magnetic dipole. At large distances, this ratio is constant, and the nonlinear effect manifests itself as a renormalization of the observed dipole field and its magnetic moment.

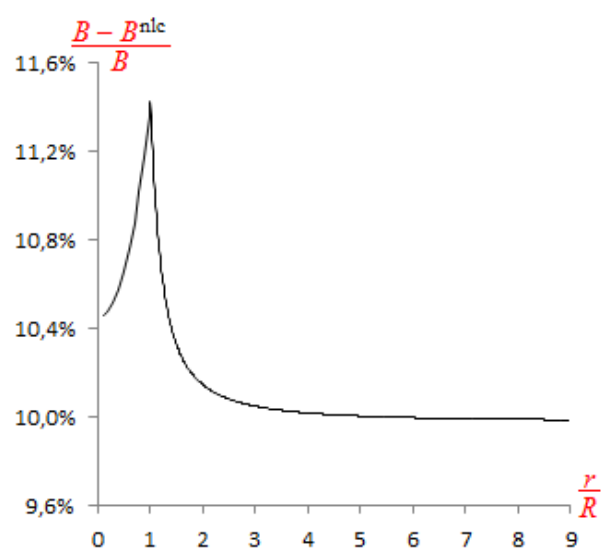

FIG 1. Nonlinear magnetic corrections to the field for Magnetars

For larger magnetization of a magnetar, which might, within the concept of its purely ferromagnetic neutron star nature, ultimately reach the magnetization of the neutron (81), we fall beyond the applicability of Eq. (64) - (70), as discussed in connection with (82) above. 


\section{Conclusion}

In this paper we considered nonlinear Maxwell equations (4), (91) - (10) in QED and other nonlinear Abelian gauge theories without external field by including the nonlinearity to the third power with respect to the field. The nonlinearity was taken into account within the low-frequency, low-momentum approximation for the off-shell four-prong photon diagram, to which end the 4-rank polarization tensor (13), (14) (also in external field (85)) is calculated as the fourh derivative over the 4-potential of the effective action taken in the local approximation, i.e. the one, where its possible dependence upon the space-time derivatives of the field strength is neglected. All the results are expressed in terms of the second derivatives of the local effective action over the two field invariants. In QED the role of this effective action is played in our calculations by the one-loop Euler-Heisenberg action.

The nonlinearly-induced current (18) to be treated as a source in the standard Maxwell equations (27) is expressed in terms of the field strengths as (23). The field equations are written separately for the special cases when a static electric (34), (30) or static magnetic (40), (37) field is present alone. The projection operators $\frac{\nabla_{i} \nabla_{j}}{\nabla^{2}}$ and $\delta_{i j}-\frac{\nabla_{i} \nabla_{j}}{\nabla^{2}}$ involved in these cubic integro-differential equations become trivial, when higher symmetries are prescribed to the solutions. Such are the cases of the electric field produced by a sperically symmetric charge distribution, charged finite-thickness plane and straight-linear thread, and the case of the magnetic field produced by a straight current-carrying wire. The above cubic equations, when treated perturbatively, give nonlinear corrections to the corresponding fields, (45), (48), (51), (56). For a lower, cylindric symmetry of the field produced by the homogeneously magnetized sphere (57), and by homogeneously electrically polarized sphere (71), the no longer trivial projection operators are realized using spherical harmonic expansion to lead to the following results: the primary dipole electric and magnetic fields reproduce themselves at large distances after the nonlinearity is switched on, the electric and magnetic moments undergoing nonlinear renormalizations. Equations for self-coupling of electric and magnetic dipole moments are (78) and (69), respectively. No spontaneous electrization, nor magnetization occurs.

Numerical estimates in QED witness that the magnetic field due to the magnetic moment of the neutron is to large at its surface to be treated in the framework of the power expansion with respect to the field. On the contrary, magnetic moments of the excited baryon $\Xi^{* 0}$ and the $\Delta^{0}$ decuplet member do fit the cubic approximation, and the corresponding nonlinear corrections to them lie within the limits admitted by the theoretical and experimental indeterminacies.

In pulsars and magnetars the relative nonlinear corrections to their magnetic moments do not exceed magnitudes admitted by the validity of the cubic approximation as long as the surface magnetic field remains below $1.6 \cdot 10^{15} \mathrm{G}$ (in Gauss units). Whereas the nonlinearity just renormalizes the value of the surface magnetic field estimated by an Earth observer, it causes the difference with the "unrenormalized" local value that may be obtained using certain theoretical models about the origin of the magnetic field of a neutron star. The absolute difference may achieve the huge value of up to $\cdot 10^{14} \mathrm{G}$ - not going beyond the approximation. Surely, many physical processes in a magnetic field are sensitive to such changes.

\section{Acknowledgements}

C. Costa acknowledges the support of CAPES. D. Gitman thanks FAPESP and CNPq for permanent support and is grateful for the support from the Project 2.3684.2011 of Tomsk State University and FTP, contract No 14.B37.21.0911. A. Shabad acknowledges the support of FAPESP, Processo 2011/51867-9, and of RFBR under the Project 11-02-00685-a. He also thanks USP for kind hospitality extended to him during his stay in Sao Paulo, Brazil, where this work was partially fulfilled. The authors are thankful to T. Adorno for some important discussions.

\section{Appendix 1}

Here we list the variational derivatives of the effective action $\Gamma$ (1) assuming that the Lagrangian $\mathfrak{L}$ does not depend upon derivatives of the field strengths. All the equations below are understood as reduced to a space- and time-independent background field $F_{\alpha \beta} \longrightarrow \mathcal{F}_{\alpha \beta}$.

The second-order derivative is:

$$
\begin{aligned}
& \frac{\delta^{2} \Gamma}{\delta A^{\mu}(x) \delta A^{\tau}(y)}=\int \mathrm{d}^{4} z\left\{\frac{\partial \mathfrak{L}}{\partial \mathfrak{F}}\left(\eta_{\mu \tau} \eta_{\alpha \beta}-\eta_{\mu \beta} \eta_{\alpha \tau}\right)+\right. \\
& +\frac{\partial \mathfrak{L}}{\partial \mathfrak{G}} \epsilon_{\alpha \mu \beta \tau}+\frac{\partial^{2} \mathfrak{L}}{\partial \mathfrak{F}^{2}} F_{\alpha \mu} F_{\beta \tau}+\frac{\partial^{2} \mathfrak{L}}{\partial \mathfrak{G}^{2}} \tilde{F}_{\alpha \mu} \tilde{F}_{\beta \tau}+ \\
& \left.+\frac{\partial^{2} \mathfrak{L}}{\partial \mathfrak{F} \partial \mathfrak{G}}\left[F_{\alpha \mu} \tilde{F}_{\beta \tau}+\tilde{F}_{\alpha \mu} F_{\beta \tau}\right]\right\}\left(\frac{\partial}{\partial z_{\alpha}} \delta^{4}(x-z)\right)\left(\frac{\partial}{\partial z_{\beta}} \delta^{4}(y-z)\right) .
\end{aligned}
$$


The third order-derivative is (note that the $F_{\gamma \sigma}$ tensor, which gives zero in the no-background field case dealt with in the body of the paper, appears in every term):

$$
\begin{aligned}
& \frac{\delta^{3} \Gamma}{\delta A^{\mu}(x) \delta A^{\tau}(y) \delta A^{\sigma}(u)}= \\
& \int \mathrm{d}^{4} z\left\{\left(\eta_{\mu \tau} \eta_{\alpha \beta}-\eta_{\mu \beta} \eta_{\alpha \tau}\right)\left[\frac{\partial^{2} \mathfrak{L}}{\partial \mathfrak{F}^{2}} F_{\gamma \sigma}+\frac{\partial^{2} \mathfrak{L}}{\partial \mathfrak{G} \partial \mathfrak{F}} \tilde{F}_{\gamma \sigma}\right] \frac{\partial}{\partial z_{\gamma}} \delta^{4}(u-z)+\right. \\
& +\epsilon_{\alpha \mu \beta \tau}\left[\frac{\partial^{2} \mathfrak{L}}{\partial \mathfrak{F} \partial \mathfrak{G}} F_{\gamma \sigma}+\frac{\partial^{2} \mathfrak{L}}{\partial \mathfrak{G}^{2}} \tilde{F}_{\gamma \sigma}\right] \frac{\partial}{\partial z_{\gamma}} \delta^{4}(u-z) \\
& +\left[\frac{\partial^{3} \mathfrak{L}}{\partial \mathfrak{F}^{3}} F_{\alpha \mu} F_{\beta \tau} F_{\gamma \sigma}+\frac{\partial^{3} \mathfrak{L}}{\partial \mathfrak{G} \partial \mathfrak{F}^{2}} F_{\alpha \mu} F_{\beta \tau} \tilde{F}_{\gamma \sigma}\right] \frac{\partial}{\partial z_{\gamma}} \delta^{4}(u-z)+ \\
& +\frac{\partial^{2} \mathfrak{L}}{\partial \mathfrak{F}^{2}}\left[F_{\alpha \mu}\left(\eta_{\tau \sigma} \frac{\partial}{\partial z^{\beta}}-\eta_{\beta \sigma} \frac{\partial}{\partial z^{\tau}}\right)+F_{\beta \tau}\left(\eta_{\mu \sigma} \frac{\partial}{\partial z^{\alpha}}-\eta_{\alpha \sigma} \frac{\partial}{\partial z^{\mu}}\right)\right] \delta^{4}(u-z)+ \\
& +\tilde{F}_{\alpha \mu} \tilde{F}_{\beta \tau}\left[\frac{\partial^{3} \mathfrak{L}}{\partial \mathfrak{F} \partial \mathfrak{G}^{2}} F_{\gamma \sigma}+\frac{\partial^{3} \mathfrak{L}}{\partial \mathfrak{G}^{3}} \tilde{F}_{\gamma \sigma}\right] \frac{\partial}{\partial z_{\gamma}} \delta^{4}(u-z)+ \\
& +\frac{\partial^{2} \mathfrak{L}}{\partial \mathfrak{G}^{2}}\left[\tilde{F}_{\alpha \mu} \epsilon_{\beta \tau \gamma \sigma}+\tilde{F}_{\beta \tau} \epsilon_{\alpha \mu \gamma \sigma}\right] \frac{\partial}{\partial z_{\gamma}} \delta^{4}(u-z)+ \\
& +\frac{\partial^{2} \mathfrak{L}}{\partial \mathfrak{F} \partial \mathfrak{G}}\left[F_{\alpha \mu} \epsilon_{\beta \tau \gamma \sigma} \frac{\partial}{\partial z_{\gamma}}+\tilde{F}_{\beta \tau}\left(\eta_{\mu \sigma} \frac{\partial}{\partial z^{\alpha}}-\eta_{\alpha \sigma} \frac{\partial}{\partial z^{\mu}}\right)+\tilde{F}_{\alpha \mu}\left(\eta_{\tau \sigma} \frac{\partial}{\partial z^{\beta}}-\eta_{\beta \sigma} \frac{\partial}{\partial z^{\tau}}\right)+\right. \\
& \left.+F_{\beta \tau} \epsilon_{\alpha \mu \gamma \sigma} \frac{\partial}{\partial z_{\gamma}}\right] \delta^{4}(u-z)+ \\
& +\left[F_{\alpha \mu} \tilde{F}_{\beta \tau}+\tilde{F}_{\alpha \mu} F_{\beta \tau}\right]\left[\frac{\partial^{3} \mathfrak{L}}{\partial \mathfrak{F}^{2} \partial \mathfrak{G}} F_{\gamma \sigma}+\frac{\partial^{3} \mathfrak{L}}{\partial \mathfrak{F} \partial \mathfrak{G}^{2}} \tilde{F}_{\gamma \sigma}\right] \\
& \left.\frac{\partial}{\partial z_{\gamma}} \delta^{4}(u-z)\right\}\left(\frac{\partial}{\partial z_{\alpha}} \delta^{4}(x-z)\right)\left(\frac{\partial}{\partial z_{\beta}} \delta^{4}(y-z)\right) .
\end{aligned}
$$


The fourth-order polarization tensor, responsible for the first nonlinear correction in the no-external-field case is:

$$
\begin{aligned}
& \frac{\delta^{4} \Gamma}{\delta A^{\mu}(x) \delta A^{\tau}(y) \delta A^{\sigma}(u) \delta A^{\rho}(v)}= \\
& =\int \mathrm{d}^{4} z\left\{\frac { \partial ^ { 2 } \mathfrak { L } } { \partial \mathfrak { F } ^ { 2 } } \left[\left(\eta_{\alpha \lambda} \eta_{\rho \mu}-\eta_{\mu \lambda} \eta_{\alpha \rho}\right)\left(\eta_{\tau \sigma} \eta_{\beta \gamma}-\eta_{\beta \sigma} \eta_{\tau \gamma}\right)\right.\right. \\
& \left.+\left(\eta_{\beta \lambda} \eta_{\rho \tau}-\eta_{\tau \lambda} \eta_{\beta \rho}\right)\left(\eta_{\mu \sigma} \eta_{\alpha \gamma}-\eta_{\alpha \sigma} \eta_{\mu \gamma}\right)+\left(\eta_{\gamma \lambda} \eta_{\rho \sigma}-\eta_{\sigma \lambda} \eta_{\gamma \rho}\right)\left(\eta_{\mu \tau} \eta_{\alpha \beta}-\eta_{\mu \beta} \eta_{\alpha \tau}\right)\right] \\
& +\frac{\partial^{2} \mathfrak{L}}{\partial \mathfrak{F} \partial \mathfrak{G}}\left[\left(\eta_{\mu \tau} \eta_{\alpha \beta}-\eta_{\mu \beta} \eta_{\alpha \tau}\right) \epsilon_{\lambda \rho \gamma \sigma}+\left(\eta_{\gamma \lambda} \eta_{\rho \sigma}-\eta_{\sigma \lambda} \eta_{\gamma \rho}\right) \epsilon_{\alpha \mu \beta \tau}+\left(\eta_{\alpha \lambda} \eta_{\rho \mu}-\eta_{\mu \lambda} \eta_{\alpha \rho}\right) \epsilon_{\beta \tau \gamma \sigma}\right. \\
& \left.+\left(\eta_{\beta \lambda} \eta_{\rho \tau}-\eta_{\tau \lambda} \eta_{\beta \rho}\right) \epsilon_{\alpha \mu \gamma \sigma}+\left(\eta_{\mu \sigma} \eta_{\alpha \gamma}-\eta_{\alpha \sigma} \eta_{\mu \sigma}\right) \epsilon_{\lambda \rho \beta \tau}+\left(\eta_{\tau \sigma} \eta_{\beta \gamma}-\eta_{\beta \sigma} \eta_{\tau \gamma}\right) \epsilon_{\lambda \rho \alpha \mu}\right] \\
& +\frac{\partial^{2} \mathfrak{L}}{\partial \mathfrak{G}^{2}}\left[\epsilon_{\alpha \mu \beta \tau} \epsilon_{\lambda \rho \gamma \sigma}+\epsilon_{\lambda \rho \alpha \mu} \epsilon_{\beta \tau \gamma \sigma}+\epsilon_{\lambda \rho \beta \tau} \epsilon_{\alpha \mu \gamma \sigma}\right] \\
& +\frac{\partial^{3} \mathfrak{L}}{\partial \mathfrak{F}^{3}}\left[F_{\alpha \mu} F_{\beta \tau}\left(\eta_{\gamma \lambda} \eta_{\rho \sigma}-\eta_{\sigma \lambda} \eta_{\gamma \rho}\right)+F_{\beta \tau} F_{\gamma \sigma}\left(\eta_{\alpha \lambda} \eta_{\rho \mu}-\eta_{\mu \lambda} \eta_{\alpha \rho}\right)+F_{\alpha \mu} F_{\gamma \sigma}\left(\eta_{\beta \lambda} \eta_{\rho \tau}-\eta_{\tau \lambda} \eta_{\beta \rho}\right)\right. \\
& \left.+F_{\alpha \mu} F_{\lambda \rho}\left(\eta_{\tau \sigma} \eta_{\beta \gamma}-\eta_{\beta \sigma} \eta_{\tau \gamma}\right)+F_{\beta \tau} F_{\lambda \rho}\left(\eta_{\mu \sigma} \eta_{\alpha \gamma}-\eta_{\alpha \sigma} \eta_{\mu \gamma}\right)+F_{\gamma \sigma} F_{\lambda \rho}\left(\eta_{\mu \tau} \eta_{\alpha \beta}-\eta_{\mu \beta} \eta_{\alpha \tau}\right)\right] \\
& +\frac{\partial^{3} \mathfrak{L}}{\partial \mathfrak{F}^{2} \partial \mathfrak{G}}\left[\left(F_{\alpha \mu} \tilde{F}_{\beta \tau}+\tilde{F}_{\alpha \mu} F_{\beta \tau}\right)\left(\eta_{\gamma \lambda} \eta_{\rho \sigma}-\eta_{\sigma \lambda} \eta_{\gamma \rho}\right)+\left(F_{\beta \tau} \tilde{F}_{\gamma \sigma}+F_{\gamma \sigma} \tilde{F}_{\beta \tau}\right)\left(\eta_{\alpha \lambda} \eta_{\rho \mu}-\eta_{\mu \lambda} \eta_{\alpha \rho}\right)\right. \\
& +\left(F_{\gamma \sigma} \tilde{F}_{\lambda \rho}+\tilde{F}_{\gamma \sigma} F_{\lambda \rho}\right)\left(\eta_{\mu \tau} \eta_{\alpha \beta}-\eta_{\mu \beta} \eta_{\alpha \tau}\right)+\left(F_{\alpha \mu} \tilde{F}_{\gamma \sigma}+F_{\gamma \sigma} \tilde{F}_{\alpha \mu}\right)\left(\eta_{\beta \lambda} \eta_{\rho \tau}-\eta_{\tau \lambda} \eta_{\beta \rho}\right) \\
& +F_{\alpha \mu} \tilde{F}_{\lambda \rho}\left(\eta_{\tau \sigma} \eta_{\beta \gamma}-\eta_{\beta \sigma} \eta_{\tau \gamma}\right)+F_{\beta \tau} \tilde{F}_{\lambda \rho}\left(\eta_{\mu \sigma} \eta_{\alpha \gamma}-\eta_{\alpha \sigma} \eta_{\mu \gamma}\right) \\
& \left.+F_{\alpha \mu} F_{\beta \tau} \epsilon_{\lambda \rho \gamma \sigma}+F_{\beta \tau} F_{\gamma \sigma} \epsilon_{\lambda \rho \alpha \mu}+F_{\gamma \sigma} F_{\lambda \rho} \epsilon_{\alpha \mu \beta \tau}+F_{\alpha \mu} \epsilon_{\lambda \rho \beta \tau}\right] \\
& +\frac{\partial^{3} \mathfrak{L}}{\partial \mathfrak{F} \partial \mathfrak{G}^{2}}\left[\tilde{F}_{\alpha \mu} \tilde{F}_{\beta \tau}\left(\eta_{\gamma \lambda} \eta_{\rho \sigma}-\eta_{\sigma \lambda} \eta_{\gamma \rho}\right)+\tilde{F}_{\alpha \mu} \tilde{F}_{\lambda \rho}\left(\eta_{\tau \sigma} \eta_{\beta \gamma}-\eta_{\beta \sigma} \eta_{\tau \gamma}\right)\right. \\
& +\tilde{F}_{\beta \tau} \tilde{F}_{\lambda \rho}\left(\eta_{\mu \sigma} \eta_{\alpha \gamma}-\eta_{\alpha \sigma} \eta_{\mu \sigma}\right)+\tilde{F}_{\gamma \sigma} \tilde{F}_{\lambda \rho}\left(\eta_{\mu \tau} \eta_{\alpha \beta}-\eta_{\mu \beta} \eta_{\alpha \tau}\right)+\left(F_{\gamma \sigma} \tilde{F}_{\lambda \rho}+\tilde{F}_{\gamma \sigma} F_{\lambda \rho}\right) \epsilon_{\alpha \mu \beta \tau} \\
& \left.+F_{\gamma \sigma} \tilde{F}_{\alpha \mu} \epsilon_{\lambda \rho \beta \tau}+F_{\gamma \sigma} \tilde{F}_{\beta \tau} \epsilon_{\lambda \rho \alpha \mu}+F_{\alpha \mu} \tilde{F}_{\lambda \rho} \epsilon_{\beta \tau \gamma \sigma}+F_{\beta \tau} \tilde{F}_{\lambda \rho} \epsilon_{\alpha \mu \gamma \sigma}\right]+\frac{\partial^{4} \mathfrak{L}}{\partial \mathfrak{F}^{4}}\left[F_{\alpha \mu} F_{\beta \tau} F_{\gamma \sigma} F_{\lambda \rho}\right] \\
& +\frac{\partial^{4} \mathfrak{L}}{\partial \mathfrak{F}^{3} \partial \mathfrak{G}}\left[F_{\alpha \mu} F_{\beta \tau} F_{\gamma \sigma} \tilde{F}_{\lambda \rho}+F_{\alpha \mu} F_{\beta \tau} F_{\lambda \rho} \tilde{F}_{\gamma \sigma}+F_{\alpha \mu} F_{\gamma \sigma} F_{\lambda \rho} \tilde{F}_{\beta \tau}+F_{\beta \tau} F_{\gamma \sigma} F_{\lambda \rho} \tilde{F}_{\alpha \mu}\right] \\
& +\frac{\partial^{4} \mathfrak{L}}{\partial \mathfrak{F}^{2} \partial \mathfrak{G}^{2}}\left[F_{\gamma \sigma} F_{\lambda \rho} \tilde{F}_{\alpha \mu} \tilde{F}_{\beta \tau}+F_{\alpha \mu} F_{\beta \tau} \tilde{F}_{\gamma \sigma} \tilde{F}_{\lambda \rho}+F_{\alpha \mu} F_{\gamma \sigma} \tilde{F}_{\beta \tau} \tilde{F}_{\lambda \rho}\right. \\
& \left.+F_{\alpha \mu} F_{\lambda \rho} \tilde{F}_{\gamma \sigma} \tilde{F}_{\beta \tau}+F_{\beta \tau} F_{\lambda \rho} \tilde{F}_{\gamma \sigma} \tilde{F}_{\alpha \mu}+F_{\gamma \sigma} F_{\beta \tau} \tilde{F}_{\alpha \mu} \tilde{F}_{\lambda \rho}\right] \\
& +\frac{\partial^{4} \mathfrak{L}}{\partial \mathfrak{F} \partial \mathfrak{G}^{3}}\left[F_{\alpha \mu} \tilde{F}_{\beta \tau} \tilde{F}_{\gamma \sigma} \tilde{F}_{\lambda \rho}+F_{\beta \tau} \tilde{F}_{\alpha \mu} \tilde{F}_{\gamma \sigma} \tilde{F}_{\lambda \rho}+F_{\gamma \sigma} \tilde{F}_{\alpha \mu} \tilde{F}_{\beta \tau} \tilde{F}_{\lambda \rho}+F_{\lambda \rho} \tilde{F}_{\alpha \mu} \tilde{F}_{\beta \tau} \tilde{F}_{\gamma \sigma}\right] \\
& \left.+\frac{\partial^{4} \mathfrak{L}}{\partial \mathfrak{G}^{4}} \tilde{F}_{\alpha \mu} \tilde{F}_{\beta \tau} \tilde{F}_{\gamma \sigma} \tilde{F}_{\lambda \rho}\right\} \frac{\partial \delta^{4}(x-z)}{\partial z_{\alpha}} \frac{\partial \delta^{4}(y-z)}{\partial z_{\beta}} \frac{\partial \delta^{4}(u-z)}{\partial z_{\gamma}} \frac{\partial \delta^{4}(v-z)}{\partial z_{\lambda}} .
\end{aligned}
$$

\section{Appendix 2}

Here we continue the calculation of nonlinear correction to the magnetic dipole field starting with Eq. (62). Write the divergence of (62) in terms of the spherical harmonics $Y_{1}^{0}(\Omega)=\frac{1}{2} \sqrt{\frac{3}{\pi}} \cos \theta$ and $Y_{3}^{0}(\Omega)=\frac{1}{4} \sqrt{\frac{7}{\pi}}\left(5 \cos ^{3} \theta-3 \cos \theta\right)$ as

$$
\begin{aligned}
& \frac{-108}{\mathfrak{L}_{\mathfrak{F} \mathfrak{F}} M^{3}} \boldsymbol{\nabla} \cdot \mathfrak{h}(\mathbf{r})=-16 \delta(r-R) \sqrt{\frac{\pi}{3}} Y_{1}^{0}(\Omega) \\
& +\frac{R^{9}}{r^{9}} \delta(r-R)\left(\frac{56}{5} \sqrt{\frac{\pi}{3}} Y_{1}^{0}(\Omega)+\frac{24}{5} \sqrt{\frac{\pi}{7}} Y_{3}^{0}(\Omega)\right) \\
& +\frac{R^{9}}{r^{10}} \Theta(r-R)\left(-72 \sqrt{\frac{\pi}{3}} Y_{1}^{0}(\Omega)-24 \sqrt{\frac{\pi}{7}} Y_{3}^{0}(\Omega)\right) .
\end{aligned}
$$

We separate variables to radial and angle (normalized spherical harmonics) parts

$$
\frac{-108}{\mathfrak{L}_{\mathfrak{F} \mathfrak{F}} M^{3}} \nabla \cdot \mathfrak{h}(\mathbf{r})=\sum_{j} R_{j}(r) Y_{j}^{0}(\Omega),
$$


where, using the orthogonality relation $\int Y_{j}^{0}(\Omega) Y_{l}^{* m}(\Omega) d \Omega=\delta_{j l} \delta_{0 m}$ we get

$$
R_{j}(r)=\frac{-108}{\mathfrak{L}_{\mathfrak{F} \mathfrak{F}} M^{3}} \int \nabla \cdot \mathfrak{h}(\mathbf{r}) Y_{j}^{0}(\Omega) \mathrm{d} \Omega .
$$

With the use of (86) we obtain

$$
\begin{aligned}
& R_{j}(r)=\left[\left(-16+\frac{56}{5} \frac{R^{9}}{r^{9}}\right) \delta(r-R)-72 \frac{R^{9}}{r^{10}} \Theta(r-R)\right] \sqrt{\frac{\pi}{3}} \delta_{1 j} \\
& +\left[\frac{24}{5} \frac{R^{9}}{r^{9}} \delta(r-R)-24 \frac{R^{9}}{r^{10}} \Theta(r-R)\right] \sqrt{\frac{\pi}{7}} \delta_{3 j} .
\end{aligned}
$$

We write the Green function in the following way [29]:

$$
\frac{1}{\left|\mathbf{r}-\mathbf{r}^{\prime}\right|}=\sum_{l=0}^{\infty} \sum_{m=-l}^{l} \frac{4 \pi}{2 l+1} Y_{l}^{m}(\Omega) Y_{l}^{* m}\left(\Omega^{\prime}\right)\left\{\begin{array}{ll}
\frac{r^{\prime l}}{r^{l+1}}, & \text { if } r>r^{\prime} \\
\frac{r^{l}}{r^{\prime l+1}}, & \text { if } r<r^{\prime}
\end{array} .\right.
$$

Let us calculate (41), using (37), (87), (89) and (90):

One can separate the integrals in the two regions of the space

$$
\begin{aligned}
& \frac{-2(4 \pi)^{3} R^{9}}{\mathfrak{L}_{\mathfrak{F} \mathfrak{F}} \mathcal{M}^{3}} \mathbf{B}^{\mathrm{nl}}(\mathbf{r})=\frac{-2(4 \pi)^{3} R^{9}}{\mathfrak{L}_{\mathfrak{F} \mathfrak{F}} \mathcal{M}^{3}} \mathfrak{h}(\mathbf{r}) \\
& +\frac{1}{4 \pi} \nabla \int\left[\int_{0}^{r} \sum_{j} R_{j}\left(r^{\prime}\right) Y_{j}^{0}\left(\Omega^{\prime}\right)\left(\sum_{l=0}^{\infty} \sum_{m=-l}^{l} \frac{4 \pi}{2 l+1} Y_{l}^{m}(\Omega) Y_{l}^{* m}\left(\Omega^{\prime}\right) \frac{r^{\prime l}}{r^{l+1}}\right) r^{\prime 2} \mathrm{~d} r^{\prime} .\right. \\
& \left.+\int_{r}^{\infty} \sum_{j} R_{j}\left(r^{\prime}\right) Y_{j}^{0}\left(\Omega^{\prime}\right)\left(\sum_{l=0}^{\infty} \sum_{m=-l}^{l} \frac{4 \pi}{2 l+1} Y_{l}^{m}(\Omega) Y_{l}^{* m}\left(\Omega^{\prime}\right) \frac{r^{l}}{r^{\prime l+1}}\right) r^{\prime 2} \mathrm{~d} r^{\prime}\right] \mathrm{d} \Omega^{\prime} .
\end{aligned}
$$

Therefore, integrating over the solid angle we obtain

$$
\begin{aligned}
& \frac{-2(4 \pi)^{3} R^{9}}{\mathfrak{L}_{\mathfrak{F} \mathfrak{F}} \mathcal{M}^{3}} \mathbf{B}^{\mathrm{nl}}(\mathbf{r})=\frac{-2(4 \pi)^{3} R^{9}}{\mathfrak{L}_{\mathfrak{F} \mathfrak{F}} \mathcal{M}^{3}} \mathfrak{h}(\mathbf{r}) \\
& +\sum_{j} \frac{1}{2 j+1} \nabla\left(\int_{0}^{r} R_{j}\left(r^{\prime}\right) \frac{r^{\prime j+2}}{r^{j+1}} \mathrm{~d} r^{\prime}+\int_{r}^{\infty} R_{j}\left(r^{\prime}\right) \frac{r^{j}}{r^{\prime j-1}} \mathrm{~d} r^{\prime}\right) Y_{j}^{0}(\Omega) .
\end{aligned}
$$

Now we show the gradient in spherical coordinates

$$
\begin{aligned}
& \frac{-2(4 \pi)^{3} R^{9}}{\mathfrak{L}_{\mathfrak{F} \mathfrak{F}} \mathcal{M}^{3}} \mathbf{B}^{\mathrm{nl}}(\mathbf{r})=\frac{-2(4 \pi)^{3} R^{9}}{\mathfrak{L}_{\mathfrak{F} \mathfrak{F}} \mathcal{M}^{3}} \mathfrak{h}(\mathbf{r})+ \\
& +\sum_{j} \frac{1}{2 j+1}\left\{\frac{\partial}{\partial r}\left[\int_{0}^{r} R_{j}\left(r^{\prime}\right) \frac{r^{\prime j+2}}{r^{j+1}} \mathrm{~d} r^{\prime}+\int_{r}^{\infty} R_{j}\left(r^{\prime}\right) \frac{r^{j}}{r^{\prime j-1}} \mathrm{~d} r^{\prime} Y_{j}^{0}(\Omega)\right] \mathbf{e}_{r}\right. \\
& \left.+\frac{1}{r}\left[\int_{0}^{r} R_{j}\left(r^{\prime}\right) \frac{r^{\prime j+2}}{r^{j+1}} \mathrm{~d} r^{\prime}+\int_{r}^{\infty} R_{j}\left(r^{\prime}\right) \frac{r^{j}}{r^{\prime j-1}} \mathrm{~d} r^{\prime} \frac{\partial}{\partial \theta} Y_{j}^{0}(\Omega)\right] \mathbf{e}_{\theta}\right\} .
\end{aligned}
$$

Now we act by the derivatives

$$
\begin{aligned}
& \frac{-2(4 \pi)^{3} R^{9}}{\mathfrak{L}_{\mathfrak{F} \mathfrak{F}} \mathcal{M}^{3}} \mathbf{B}^{\mathrm{nl}}(\mathbf{r})=\frac{-2(4 \pi)^{3} R^{9}}{\mathfrak{L}_{\mathfrak{F} \mathfrak{F}} \mathcal{M}^{3}} \mathfrak{h}(\mathbf{r}) \\
& +\sum_{j} \frac{1}{2 j+1}\left[\Sigma_{j}(r) Y_{j}^{0}(\Omega) \mathbf{e}_{r}+\Xi_{j}(r) \frac{\partial}{\partial \theta} Y_{j}^{0}(\Omega) \mathbf{e}_{\theta}\right] .
\end{aligned}
$$

where

$$
\Sigma_{j}(r)=-(j+1) \int_{0}^{r} R_{j}\left(r^{\prime}\right) \frac{r^{j+2}}{r^{j+2}} \mathrm{~d} r^{\prime}+j \int_{r}^{\infty} R_{j}\left(r^{\prime}\right) \frac{r^{j-1}}{r^{\prime j-1}} \mathrm{~d} r^{\prime},
$$

and

$$
\Xi_{j}(r)=\int_{0}^{r} R_{j}\left(r^{\prime}\right) \frac{r^{\prime j+2}}{r^{j+2}} \mathrm{~d} r^{\prime}+\int_{r}^{\infty} R_{j}\left(r^{\prime}\right) \frac{r^{j-1}}{r^{\prime j-1}} \mathrm{~d} r^{\prime} .
$$


Therefore, by (89)

$$
\begin{aligned}
& \Sigma_{j}(r)=\sqrt{\frac{\pi}{3}}\left[-\frac{64}{5} \delta_{1 j}+\frac{432}{55} \frac{r^{2}}{R^{2}} \delta_{3 j}\right] \Theta(R-r) \\
& +\sqrt{\frac{\pi}{3}}\left[\left(\frac{168}{5} \frac{R^{3}}{r^{3}}-32 \frac{R^{9}}{r^{9}}\right) \delta_{1 j}+\left(\frac{24}{5} \frac{R^{5}}{r^{5}}-\frac{336}{11} \frac{R^{9}}{r^{9}}\right) \delta_{3 j}\right] \Theta(r-R),
\end{aligned}
$$

and

$$
\begin{aligned}
& \Xi_{j}(r)=\sqrt{\frac{\pi}{7}}\left[-\frac{64}{5} \delta_{1 j}+\frac{144}{55} \frac{r^{2}}{R^{2}} \delta_{3 j}\right] \Theta(R-r) \\
& +\sqrt{\frac{\pi}{7}}\left[\left(-\frac{84}{5} \frac{R^{3}}{r^{3}}+4 \frac{R^{9}}{r^{9}}\right) \delta_{1 j}+\left(-\frac{6}{5} \frac{R^{5}}{r^{5}}+\frac{42}{11} \frac{R^{9}}{r^{9}}\right) \delta_{3 j}\right] \Theta(r-R) .
\end{aligned}
$$

Substituting (62) and (93), (94) in (91) we obtain the nonlinear magnetic field (63).

\section{Appendix 3}

Here we continue the calculation of nonlinear correction to the electric dipole field starting with Eq. (74).

The electric field of an electric dipole can be dealt with analogously to the case of magnetic dipole, although we use the longitudinal projection instead the transverse projection. The electric field (72) can be also written as

$$
\mathbf{E}^{\operatorname{lin}}(\mathbf{r})=-\frac{p}{4 \pi R^{3}} \Theta(R-r) \mathbf{e}_{3}+\left(\frac{3 p}{4 \pi r^{3}} \cos \theta \mathbf{e}_{r}-\frac{p}{4 \pi r^{3}} \mathbf{e}_{3}\right) \Theta(r-R),
$$

Let us calculate (30) substituting $\mathbf{E}^{\operatorname{lin}}(\mathbf{r})$ for $\mathbf{E}(\mathbf{r})$.

The product $\frac{\left(4 \pi R^{3}\right)^{3}}{p^{3}} \mathbf{E}(\mathbf{r}) E^{2}(\mathbf{r})$ is given by

$$
\begin{aligned}
& -\frac{2(4 \pi)^{3} R^{9}}{\mathfrak{L}_{\mathfrak{F} \mathfrak{F}} p^{3}} \mathcal{E}(\mathbf{r})=\left(-\cos \theta \mathbf{e}_{r}+\sin \theta \mathbf{e}_{\theta}\right) \Theta(R-r) \\
& +\left[\left(2 \frac{R^{9}}{r^{9}} \cos \theta+6 \frac{R^{9}}{r^{9}} \cos ^{3} \theta\right) \mathbf{e}_{r}+\left(4 \frac{R^{9}}{r^{9}} \sin \theta-3 \frac{R^{9}}{r^{9}} \sin ^{3} \theta\right) \mathbf{e}_{\theta}\right] \Theta(r-R) .
\end{aligned}
$$

By (62), one can compare $\mathcal{E}(\mathbf{r})$ to $\mathfrak{h}(\mathbf{r})$

$$
\frac{2(4 \pi)^{3} R^{9}}{\mathfrak{L}_{\mathfrak{F} \mathfrak{F}} p^{3}} \mathcal{E}(\mathbf{r})=\frac{2(4 \pi)^{3} R^{9}}{\mathfrak{L}_{\mathfrak{F} \mathfrak{F}} \mathcal{M}^{3}} \mathfrak{h}(\mathbf{r})-9 \mathbf{e}_{3} \Theta(R-r) .
$$

The electric field is calculated from (34) and (97)

$$
\begin{aligned}
& -\frac{2(4 \pi)^{3} R^{9}}{\mathfrak{L}_{\mathfrak{F} \mathfrak{F}} p^{3}} \mathbf{E}^{\mathrm{nl}}(\mathbf{r})=-\frac{1}{4 \pi} \boldsymbol{\nabla} \int \frac{\boldsymbol{\nabla}^{\prime} \cdot\left[\frac{2(4 \pi)^{3} R^{9}}{\mathfrak{L}_{\mathfrak{F} \mathfrak{F}} p^{3}} \mathcal{E}\left(\mathbf{r}^{\prime}\right)\right]}{\left|\mathbf{r}-\mathbf{r}^{\prime}\right|} \mathrm{d} \mathbf{r}^{\prime} \\
& =-\frac{2(4 \pi)^{3} R^{9}}{\mathfrak{L}_{\mathfrak{F} \mathfrak{F}} \mathcal{M}^{3}}\left(\mathbf{B}^{\mathrm{nl}}(\mathbf{r})-\mathfrak{h}(\mathbf{r})\right)+\frac{1}{4 \pi} \boldsymbol{\nabla} \int \frac{\boldsymbol{\nabla}^{\prime} \cdot\left[9 \mathbf{e}_{3} \Theta\left(R-r^{\prime}\right)\right]}{\left|\mathbf{r}-\mathbf{r}^{\prime}\right|} \mathrm{d} \mathbf{r}^{\prime}
\end{aligned}
$$

It is easy to show that

$$
\boldsymbol{\nabla} \cdot\left[9 \mathbf{e}_{3} \Theta(R-r)\right]=-9 \delta(r-R) \cos \theta
$$

The second term of (99) can be calculated in the same way as in the magnetic case, i.e., by expanding of the Green function in spherical harmonics, and calculating the solid angle integral with the help of orthogonality relations

$$
\begin{aligned}
& -9 \frac{1}{4 \pi} \nabla \int \frac{\delta\left(R-r^{\prime}\right) \cos \theta}{\left|\mathbf{r}-\mathbf{r}^{\prime}\right|} \mathrm{d} \mathbf{r}^{\prime} \\
& =-9 \frac{1}{4 \pi} \nabla \int\left[\int_{0}^{r} \frac{\delta\left(R-r^{\prime}\right)}{\left|\mathbf{r}-\mathbf{r}^{\prime}\right|} r^{\prime 2} \mathrm{~d} r^{\prime}+\int_{r}^{\infty} \frac{\delta\left(R-r^{\prime}\right)}{\left|\mathbf{r}-\mathbf{r}^{\prime}\right|} r^{\prime 2} \mathrm{~d} r^{\prime}\right] \frac{1}{2} \sqrt{\frac{\pi}{3}} Y_{1}^{0}\left(\Omega^{\prime}\right) \mathrm{d} \Omega^{\prime} \\
& =-3 \nabla\left[\left(\int_{0}^{r} \delta\left(R-r^{\prime}\right) \frac{r^{\prime 3}}{r^{2}} \mathrm{~d} r^{\prime}+\int_{r}^{\infty} \delta\left(R-r^{\prime}\right) r \mathrm{~d} r^{\prime}\right) \frac{1}{2} \sqrt{\frac{\pi}{3}} Y_{1}^{0}(\Omega)\right] \\
& =-3 \nabla\left[\left(\frac{R^{3}}{r^{2}} \Theta(r-R)+r \Theta(R-r)\right) \cos \theta\right] \\
& =\left(6 \frac{R^{3}}{r^{3}} \Theta(r-R)-3 \Theta(R-r)\right) \cos \theta \mathbf{e}_{r}+\left(3 \frac{R^{3}}{r^{3}} \Theta(r-R)+3 \Theta(R-r)\right) \sin \theta \mathbf{e}_{\theta} .
\end{aligned}
$$


Therefore,

$$
\begin{aligned}
& -\frac{2(4 \pi)^{3} R^{9}}{\mathfrak{L}_{\mathfrak{F} \mathfrak{F}} p^{3}} \mathbf{E}^{\mathrm{nl}}(\mathbf{r})=-\frac{2(4 \pi)^{3} R^{9}}{\mathfrak{L}_{\mathfrak{F} \mathfrak{F}} \mathcal{M}^{3}}\left(\mathbf{B}^{\mathrm{nl}}(\mathbf{r})-\mathfrak{h}(\mathbf{r})\right)+\left(6 \frac{R^{3}}{r^{3}} \Theta(r-R)-3 \Theta(R-r)\right) \cos \theta \mathbf{e}_{r} \\
& +\left(3 \frac{R^{3}}{r^{3}} \Theta(r-R)+3 \Theta(R-r)\right) \sin \theta \mathbf{e}_{\theta} .
\end{aligned}
$$

Thus the expression for electric field (75) is obtained.

\section{References}

[1] A.E. Shabad, Lett. Nuov. Cim. 2, 457 (1972), Ann. Phys. (N.Y.) 90, 166 (1975)

[2] A.E. Shabad and V.V. Usov, Nature 295, 215 (1982)

[3] A.E. Shabad and V.V. Usov, Astrophys. and Space Sci. 117, 309 (1985); ibid 128, 377 (1986); H. Herold, H. Ruder and G. Wunner, Phys. Rev. Lett. 54, 1452 (1985); D.J. Thompson et al., ApJ. 436, 229 (1994); V.V. Usov and D.B. Melrose, ApJ, 464, 306 (1996)

[4] A.K. Harding and D. Lai, Physics of strongly magnetized neutron stars, Rep. Prog. Phys. 69, 2631 (2006)

[5] T.Vachaspati, Phys. Lett. B265, 258(1991); K.Enquist and P.Olesen, Phys. Lett. B 319, 178 (1993); D.Grasso and H.R.Rubinstein, Phys. Rept. 348, 163 (2001)

[6] R.C.Duncan and C.Thompson, Astro. Phys. J. 392, L9 (1992); J.M.Lattimer and M.Prakash, Phys. Rept. 442, 109 (2007)

[7] R.P. Negreiros, F. Weber, M. Malheiro, V. Usov, Phys. Rev. D 80, 083006 (2009); R.P. Negreiros, I.N.

Mishustin, S. Schramm, F. Weber, Phys. Rev. D 82, 103010 (2010)

[8] D.E.Kharzeev, L.D.McLerran and H.J.Warringa, Nucl. Phys. A803, 227 (2008); V.S.Skokov, A.Illarionov and V.Toneev, Int. J. Mod. Phys. A24, 5925 (2009)

[9] Yu.A. Simonov, B.O. Kerbikov, M.A. Andreichikov, Asymptotic freedom in strong magnetic field, arXiv:1210.0227 hep-ph]; V.D. Orlovsky, V.I. Shevchenko, Towards a quantum theory of the chiral magnetic effect, Phys. Rev. D, 82, 094032 (2010); M. A. Andreichikov, V. D. Orlovsky, and Yu. A. Simonov, Quark-Antiquark System in Ultra-Intense Magnetic Field, arXiv:1211.6568 [hep-ph]; Yu.A. Simonov, Neutral 3-body system in a strong magnetic field: Factorization and exact solutions, Phys. Lett. B, 719, 464 (2013); K. Tuchin, arXiv:1301.0099 [hep-ph]

[10] V.V. Braguta, P.V. Buividovich, T. Kalaydzhyan, M.I. Polikarpov, Topological and magnetic properties of the QCD vacuum probed by overlap fermions, arXiv:1302.6458v2 [hep-lat]

[11] A. Stern, Phys. Rev. Lett. 100, 061601 (2008); T.C. Adorno, D. M. Gitman, A.E. Shabad, D.V. Vassilevich, Phys.Rev. D 84, 085031 (2011), ibid 84, 065003 (2011); T.C. Adorno, D.M. Gitman, A.E. Shabad, Phys. Rev. D 86, 027702 $(2012)$

[12] A. E. Shabad, Polarization of the vacuum and quantum relativistic gas in an external field. Nova Science Publishers, New York, (1991). [Trudy Fiz. Inst. im. P.N. Lebedeva 192, 5 (1988)].

[13] Probing the Quantum Vacuum, Edited by Dittrich, W.; Gies, H., Springer Tracts in Modern Physics, vol. 166 (2000)

[14] A. Kuznetsov and N. Micheev, Electroweak Processes in External Electromagnetic Fields, Springer Tracs in Modern Physics, vol. 197 (Springer, New York, Berlin etc., 2004)

[15] D. M. Gitman, A. E. Shabad, Nonlinear (magnetic) correction to the field of a static charge in an external field. Phys. Rev. D 86, 125028 (2012)

[16] A.I. Akhiezer and V.B. Berestetskii, Quantum Electrodynamics (NAUKA, Moscow, 1969 (in Russian); Interscience Pbls., John Wiley \& Sons, New York-London-Sydney, 1965)

[17] V.B. Berestetsky, E.M. Lifshits, and L.P. Pitayevsky, Quantum Electrodynamics (Nauka, Moscow, 1989; Pergamon Press Oxford, New York, 1982) 
[18] V. S. Beskin, A. V. Gurevich and Ya. N. Istomin, Physics of the Pulsar Magnetosphere (Cambridge, 1993)

[19] T.Tatsumi, Magnetic instability of quark matter, Phys. Lett. B489, 280 (2000); Ferromagnetic properties of quark matter - an origin of magnetic field in compact stars -, arXiv :0910.1642 [hep-ph] (2009)

[20] S. Weinberg, The Quantum Theory of Fields, (University Press, Cambridge, 2001)

[21] E.S. Fradkin, D.M. Gitman and S.M. Shvartsman, Quantum Electrodynamics with Unstable Vacuum (Springer, Berlin, 1991)

[22] A.E. Shabad and V.V. Usov, Effective Lagrangian in nonlinear electrodynamics and its properties of causality and unitarity, Phys. Rev. D 83, 105006 (2011)

[23] J. Beringer et al. (Particle Data Group), Phys. Rev. D 86, 010001 (2012) (URL: http://pdg.lbl.gov)

[24] F.X. Lee, R. Kelly, L. Zhou, W. Wilcox, Baryon magnetic moments in the background field methods, Phys.Lett. B627, 71 (2005), arXiv: 0509067 [hep-lat]

[25] M.N. Butler, M.J. Savage, and R.P. Springer, Phys. Rev. D 49, 3459 (1994), hep-ph/9308317; L. Geng, arXiv: 1301.7815 [nucl-th]

[26] R. Turolla, S. Zane, J.A. Pons, P. Esposito, N. Rea Is SGR 0418+5729 indeed a waning magnetar? arXiv:1107.5488 [astro-ph.HE]

[27] C. Kouveliotou et al., Nature (London) 393, 235 (1998); Mereghetti S. The strongest cosmic magnets: soft gamma-ray repeaters and anomalous X-ray pulsars, Astronomy and Astrophysics Review, 15, 225 (2008)

[28] V.V. Usov, Nature (London) 357, 472 (1992)

[29] G. Arfken, Mathematical methods for physicists (Miami University, Academic Press, Inc., Third edition, 2006) 